\title{
Stability and confinement of nonrelativistic sheet electron beams with periodic cusped magnetic focusing
}

\author{
John H. Booske and Brian D. McVeya) \\ Electrical and Computer Engineering Department, University of Wisconsin-Madison, Madison, \\ Wisconsin 53706 \\ Thomas M. Antonsen, Jr. \\ Laboratory for Plasma Research, Department of Electrical Engineering and Department of Physics \\ and Astronomy, University of Maryland, College Park, Maryland 20742
}

(Received 27 August 1992; accepted for publication 6 January 1993)

\begin{abstract}
Sheet electron beams focused by periodically cusped magnetic (PCM) fields are stable against low-frequency velocity-shear instabilities (such as diocotron mode). This is in contrast to more familiar unstable behavior in uniform solenoidal magnetic fields. Two rectangular-cross-section magnetic configurations capable of focusing in both transverse dimensions are investigated: (i) a closed-side two-plane PCM configuration that is topologically equivalent to conventional round-cross-section PPM focusing; and (ii) an open-side configuration that uses ponderomotive PCM focusing in the vertical plane and simple $v_{z} B_{y}$ Lorentz force focusing in the horizontal plane. Both configurations are capable of stable sheet beam confinement. The open-side configuration appears more practical both for focusing and for realizing matched (cold) beam conditions in which the beam envelope is free from oscillations. For realistic beams with finite emittance, the existence of a matched cold beam solution implies less emittance growth at beam injection.
\end{abstract}

\section{INTRODUCTION}

Sheet or ribbon electron beams have been of interest for numerous applications, ranging from microwave tubes to gas laser excitation and plasma chemistry reactors. A summary of desirable applications can be found in Ref. 1. Perhaps the most familiar (infamous) feature of sheet electron beams concerns their behavior in the presence of a uniform solenoidal focusing magnetic field. Namely, this is an unstable configuration, due to $\mathbf{E} \times \mathbf{B}$ drift forces arising from the guiding magnetic field $\mathbf{B}$ and the transverse electric space-charge fields of the beam. The tendency for lowfrequency (MHD) beam instability in this configuration can manifest itself in several ways. The simplest manifestation is a rotational mode discussed in Ref. 2. More familiar are transverse kink, diocotron, and/or filamentation instabilities. There appears to be some historical differences regarding the use of the term "diocotron," with some authors applying it to "ac space-charge" kink-type modes", and others associating it with $\mathbf{E} \times \mathbf{B}$ velocity shear effects. Antonsen and $\mathrm{Ott}^{5}$ identify that the mechanism is identical for both cases. The physical consequence of diocotron instability is that the beam forms localized kinks and vortices, with the eventual result of beam filamentation, "heating," breakup, and possibly interception on the confining vessel walls. A recent review article discussing diocotrontype instabilities is also available. ${ }^{6}$

The onset of diocotron instability for sheet beams in solenoidal magnetic fields is not universally assured. There are several methods for stabilization that have been theoretically predicted and experimentally demonstrated. First, the instability is convective, requiring a finite length of

\footnotetext{
${ }^{a)}$ On leave from Los Alamos National Laboratory, Los Alamos, NM.
}

propagation before becoming unacceptably pronounced. A worst-case (lower-bound) estimate for the diocotron instability growth length can be obtained as ${ }^{5-9}$

$$
L_{d}(\mathrm{~cm})>v_{z} \frac{\omega_{c}}{\omega_{p}^{2}} \approx 800 \gamma^{3} \beta_{z}^{2} \frac{B_{z}(\mathrm{kG})}{J_{b}\left(\mathrm{~A} / \mathrm{cm}^{2}\right)},
$$

where $\omega_{c}=e B / m$ is the electron cyclotron frequency, $\omega_{p}^{2}=n e^{2} / \gamma^{3} m \epsilon_{0}$ is the square of the relativistic beam plasma frequency ( $n$ is the beam density in the laboratory frame), $\gamma=\left(1-\beta_{z}^{2}\right)^{-1 / 2}$ is the rclativistic encrgy parameter, and $\beta_{z}=v_{z} / c$ is the beam axial velocity normalized to the speed of light in vacuum. Clearly, the diocotron instability can be suppressed by large beam energies (large $\gamma$ ) or low-beamcurrent densities $J_{b}$. This is just a consequence of stiffer beams-i.e., a space-charge instability being reduced by reducing the effective space charge. Also, strong magneticfield intensity $B_{z}$ will increase $L_{d}$, thereby suppressing the instability. This has been experimentally demonstrated in several configurations. ${ }^{2,10,11}$ Placing a sheet beam between closely spaced conducting plates (or an annular beam in close proximity to an outer conducting wall) will also significantly reduce the instability rate. ${ }^{2,3,5,9}$ This effect is associated with image charges and currents in the conducting boundaries. Finite-temperature effects ${ }^{5}$ and space-charge neutralization by ions ${ }^{4}$ are other mechanisms for diocotron instability suppression.

Several of the methods mentioned above for sheet beam stabilization are not attractive for compact microwave tubes. For example, the generation of strong (several kilogauss) solenoidal fields requires bulky electromagnets and associated power supplies. The longer the tube interaction length, the greater the field intensity required. Thus, this approach is primarily relevant to devices with short 
interaction lengths, such as oscillators. The use of relativistic beam energies is obviously restricted to relativistic accelerator or relativistic vacuum electronics applications. More conventional devices with lower beam voltages $V_{b}$ $<100 \mathrm{kV}$ must rely on keeping $J_{b}$ small to obtain stiff beams. This approach is not always practical for moderateto high-power millimeter-wave devices which require significant beam current propagated through small clearance microwave circuits (resulting in high $J_{b}$ ). Space-charge neutralization by background ions is generally incompatible with conventional devices based on thermionic electron-beam sources. An exception to this latter case involves the plasma-filled technology of the PASOTRON ${ }^{\mathrm{TM}}$, although a sheet beam has not actually been used in that particular device. ${ }^{12}$

An alternative method to obtain a stable sheet electron-beam configuration is to employ a periodicrather than uniform solenoidal-focusing magnetic field. Recently such a configuration has been studied both theoretically and experimentally. This work investigated relativistic sheet beams with periodic "wiggler" focusing. A robust stability was expected, due to a combination of "stiff beam" parameters and the fact that the ponderomotive focusing provides a stable minimum energy state against low-frequency (MHD) instabilities. This latter issue will be discussed in greater detail below. Convincing experimental evidence for this stability have included observations of very low-beam interception currents (high-beam transmission) for short, ${ }^{1}$ intermediate, ${ }^{13,14}$ and long ${ }^{15}$ wiggler magnets.

Sheet electron beams would be particularly attractive for nonrelativistic applications (beam voltages less than 50 $\mathrm{kV}$ ) such as moderate and high average power millimeterwave tubes. ${ }^{7}$ Unfortunately, the wiggler-focusing scheme becomes more problematic with low-voltage beams because the large peak magnetic fields required for focusing beam space charge impart an excessive amount of transverse "wiggling" velocity, possibly even causing complete beam deflection. An interesting alternative candidate, however, is to employ a rectangular configuration of periodic cusped magnetic (PCM) fields such as that illustrated in Fig. 1. A cylindrical configuration of periodic cusped fields using permanent magnets to focus a conventional roundcross-section beam is commonly referred to in the microwave tube industry as PPM focusing. ${ }^{16}$

The only known analysis of sheet beam stability in PCM fields is an article by Dohler ${ }^{17}$ who modified Pierce ${ }^{3}$ and Kyhl and Webster's ${ }^{4}$ analysis for a sheet beam in a uniform solenoidal field. Dohler's analysis approach, however, is only applicable to the case of a slowly varying magnetic field-specifically, for conditions where the beam plasma wavelength and the diocotron growth length are much shorter than the magnetic-field gradient scale length. For a sinusoidally varying focusing field, this amounts to the restriction $\lambda_{p}=2 \pi v_{z} / \omega_{p} \ll l_{m} / 4$, where $l_{m}$ is the magnet spatial period. However, PCM focusing is generally most effective in the limit of short magnet period, ${ }^{16} l_{m} / 4<\lambda_{p}$, where Dohler's analysis does not apply.

This article is organized as follows. Unperturbed orbits

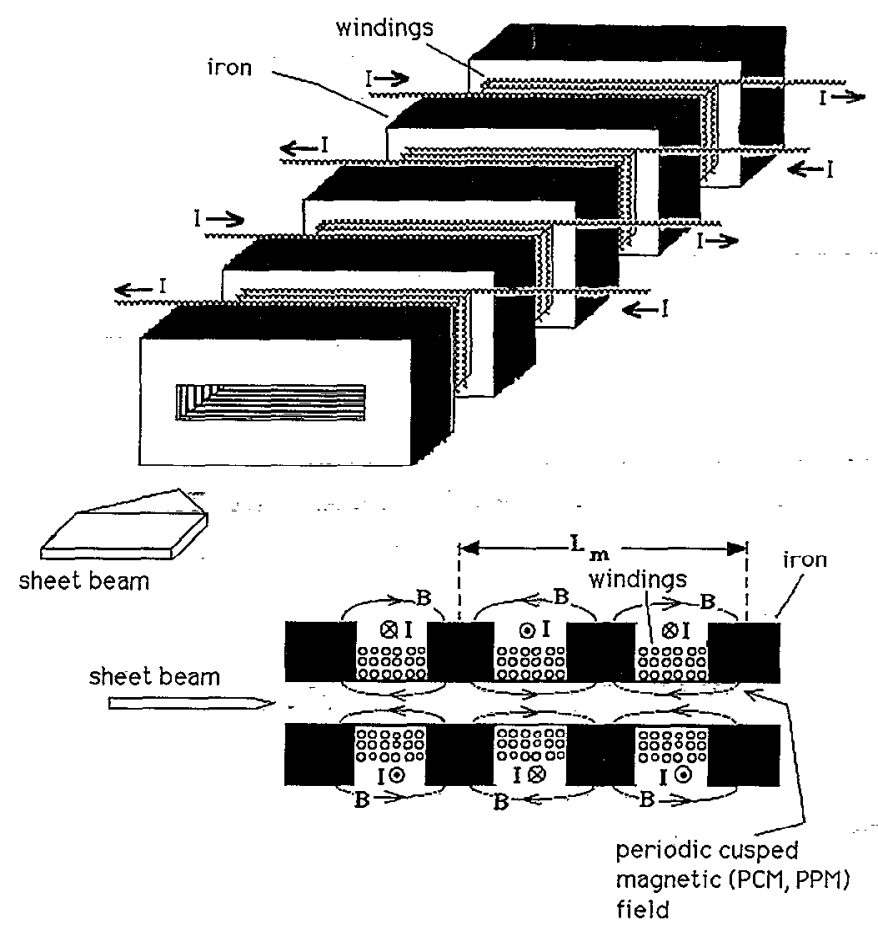

FIG. 1. Schematic of two-plane, rectangular-cross-section, PCM focusing configuration (electromagnet version).

and period-averaged magnetic focusing forces are obtained in Sec. II for electrons in two-dimensional rectangular PCM focusing. Section III is an analysis of the lowfrequency stability of nonrelativistic sheet beams in periodic magnetic focusing. Detailed formulas for magnetic and space-charge electric fields are derived in Sec. IV for a sheet electron beam in rectangular-cross-section PCM focusing. Identification of a practical two-plane-focusing PCM configuration that allows for beam matching (to minimize emittance growth) is discussed in Sec. V. Summary and Conclusions are presented in Sec. VI.

\section{UNPERTURBED ORBITS FOR ELECTRONS IN TWO-PLANE RECTANGULAR PERIODIC CUSP FOCUSING}

Consider a configuration such as that shown in Fig. 1 with rectangular cross section and periodic cusps in both transverse planes. Anticipating a result from later discussion, the near-axis fields for this configuration can be approximated by the following expressions which are derived from a scalar potential, $\mathbf{B}=-\nabla \chi_{m}$ and

$$
\begin{aligned}
\chi_{m} & =\left(B_{0} / k_{m}\right) \cosh \left(k_{x} x\right) \cosh \left(k_{y} y\right) \cos \left(k_{m} z\right): \\
B_{x} & =-\left(k_{x} / k_{m}\right) B_{0} \sinh \left(k_{x} x\right) \cosh \left(k_{y} y\right) \cos \left(k_{m} z\right) \\
& \approx-\left(k_{x}^{2} / k_{m}^{2}\right) B_{0} x \cos \left(k_{m} z\right), \\
B_{y} & =-\left(k_{y} / k_{m}\right) B_{0} \cosh \left(k_{x} x\right) \sinh \left(k_{y} y\right) \cos \left(k_{m} z\right) \\
& \approx-\left(k_{y}^{2} / k_{m}^{2}\right) B_{0} y \cos \left(k_{m} z\right),
\end{aligned}
$$

and 
$B_{z}=B_{0} \cosh \left(k_{x} x\right) \cosh \left(k_{y} y\right) \sin \left(k_{m} z\right) \approx B_{0} \sin \left(k_{m} z\right)$,

where $k_{m}^{2}=k_{x}^{2}+k_{y}^{2}$ follows from $\nabla \cdot \mathbf{B}=0$. The (nonrelativistic) Lorentz equation has the three vector components:

$$
\begin{aligned}
& \ddot{x}=\Omega_{0}\left[\dot{y}\left(B_{z} / B_{0}\right)-\dot{z}\left(B_{y} / B_{0}\right)\right], \\
& \ddot{y}=\Omega_{0}\left[\dot{z}\left(B_{x} / B_{0}\right)-\dot{x}\left(B_{z} / B_{0}\right)\right],
\end{aligned}
$$

and

$$
\ddot{z}=\Omega_{0}\left[\dot{x}\left(B_{y} / B_{0}\right)-\dot{y}\left(B_{x} / B_{0}\right)\right],
$$

where $\Omega_{0} \equiv q B_{0} / m$. For the zeroth-order motion we simply assume $\dot{z}_{0} \approx v_{b}$, where $v_{b}$ is the beam velocity. The terms

$$
\ddot{x}_{1}=-v_{b} \Omega_{0}\left(B_{y} / B_{0}\right)
$$

and

$$
\ddot{y}_{1}=v_{b} \Omega_{0}\left(B_{x} / B_{0}\right)
$$

generate first-order "wiggle" motion $\left(x_{1}, y_{1}\right)$ for electrons displaced from the axis. Substituting Eq. (2b) into Eq. (4a) and integrating yields

$$
\dot{x}_{1}=\Omega_{0}\left(k_{y}^{2} / k_{m}^{2}\right) y_{0} \sin \left(k_{m} v_{b} t\right)
$$

or

$$
x_{1}=\left(-\Omega_{0} / k_{m} v_{b}\right)\left(k_{y}^{2} / k_{m}^{2}\right) y_{0} \cos \left(k_{m} v_{b} t\right)
$$

and

$$
\dot{y}_{1}=-\Omega_{0}\left(k_{x}^{2} / k_{m}^{2}\right) x_{0} \sin \left(k_{m} v_{b} t\right)
$$

or

$$
y_{1}=\left(\Omega_{0} / k_{m} v_{b}\right)\left(k_{x}^{2} / k_{m}^{2}\right) x_{0} \cos \left(k_{m} v_{b} t\right) .
$$

We now turn to the zeroth-order motion in the $x$ and $y$ dimensions-i.e., that motion which survives averaging over a "wiggle" period. In the horizontal plane we have

$$
\begin{aligned}
\ddot{x}_{0}= & \Omega_{0}\left(\dot{y}_{1} \frac{B_{z}}{B_{0}}-v_{b} \frac{B_{y}}{B_{0}}\right) \\
= & -\Omega_{0}^{2} \frac{k_{x}^{2}}{k_{m}^{2}} x_{0} \sin ^{2}\left(k_{m} v_{b} t\right) \\
& +\Omega_{0} v_{b} \frac{k_{y}^{2}}{k_{m}^{2}}\left(y_{0}+y_{1}\right) \cos \left(k_{m} v_{b} t\right)
\end{aligned}
$$

which after period averaging simplifies to

$$
\ddot{x}_{0}=-\frac{\Omega_{0}^{2}}{2} x_{0} \frac{k_{x}^{2}}{k_{m}^{2}}\left(1-\frac{k_{y}^{2}}{k_{m}^{2}}\right),
$$

i.e., simple harmonic betatron ${ }^{18}$ motion associated with a ponderomotive focusing force:

$$
F_{x 0}=-\left[m \frac{\Omega_{0}^{2}}{2} \frac{k_{x}^{2}}{k_{m}^{2}}\left(1-\frac{k_{y}^{2}}{k_{m}^{2}}\right)\right] x_{0} .
$$

By a similar process, we obtain the zeroth-order betatron motion in the vertical $(y)$ plane,

$$
\ddot{y}_{0}=-\frac{\Omega_{0}^{2}}{2} y_{0} \frac{k_{y}^{2}}{k_{m}^{2}}\left(1-\frac{k_{x}^{2}}{k_{m}^{2}}\right),
$$

associated with the ponderomotive focusing force,

$$
F_{y 0}=-\left[m \frac{\Omega_{0}^{2}}{2} \frac{k_{y}^{2}}{k_{m}^{2}}\left(1-\frac{k_{x}^{2}}{k_{m}^{2}}\right)\right] y_{0} .
$$

\section{STABILITY OF SHEET BEAMS IN PERIODIC MAGNETIC FOCUSING}

In this section we discuss the stability of sheet electron beams in periodic magnetic systems such as wiggler or PCM focusing. The stability derives from the fact that for modes cvolving over distances longer than a magnet pcriod, the magnetic focusing forces can be accurately represented by their period-averaged, ponderomotive expressions arising from the $\mathbf{v} \times \mathbf{B}$ "beating" of an oscillatory ("wiggle") velocity against the oscillatory periodic magnetic focusing field [i.e., Eqs. (6b) and (6d)]. For a configuration such as that shown in Fig. 1 with periodic focusing in both transverse planes the (nonrelativistic) nearaxis, period-averaged $\mathbf{v} \times \mathbf{B}$ force terms can be approximated according to Eqs. (6b) and (6d). As discussed in a later section, when the configuration is much wider in the horizontal plane than in the vertical plane, one obtains $k_{y} \approx k_{m}$ and $k_{x} \approx \sqrt{k_{m}^{2}-k_{y}^{2}}$. Hence the near-axis, period-averaged ponderomotive PCM forces are approximately

$$
\left\langle F_{y}\right\rangle \approx\left(-m \Omega_{0}^{2} / 2\right) y
$$

and

$$
\left\langle F_{x}\right\rangle \approx\left(-m \Omega_{0}^{2} / 2\right) C_{x} x,
$$

where the brackets \langle\rangle denote averaging over a period and $C_{x} \approx\left(k_{x} / k_{m}\right)^{4}$. Clearly, Eqs. (7) are in the form of a potential well $U(x, y)$ with a force-free equilibrium point at the origin $(x=y=0)$ and for which the second variation of the potential is positive definite for translational perturbations of the sort $q \rightarrow q+\delta q$, i.e.,

$$
\delta^{2} U=\frac{1}{2} \frac{\partial^{2} U}{\partial q_{i} \partial q_{j}} \delta q_{i} \delta q_{j}=-\frac{1}{2} \delta q_{i} \frac{\partial\left\langle F_{j}\right\rangle}{\partial q_{i}} \delta q_{j} \geqslant 0 .
$$

Hence, in accordance with the stability criteria articulated by Gibbs, ${ }^{19}$ one can expect that such a configuration is stable against low-frequency space-charge modes. We illustrate this fact with a simplified treatment of diocotron instability patterned after that originally presented by Buneman. ${ }^{18,20}$ For reference, we briefly review the model ${ }^{20}$ for a sheet beam in a uniform solenoidal field followed by a modification of this model for the periodically focused sheet beam.

The model assumes a uniform solenoidal field $\mathbf{B}=B_{0} \hat{\mathbf{z}}$ and a thin beam with charge density per unit area $N q=n t q$ where $t$ is the beam thickness, $n$ is the electron density per unit volume, and $N$ is the electron density per unit area. Beam perturbations of the sort in Fig. 2 are considered. Focusing on the dynamics of electrons at the beam center, the equations of motion are

$$
\begin{aligned}
& \ddot{x}=\left(q E_{x} / m\right)-\Omega_{0} \dot{y}, \\
& \ddot{y}=\left(q E_{y} / m\right)+\Omega_{0} \dot{x},
\end{aligned}
$$




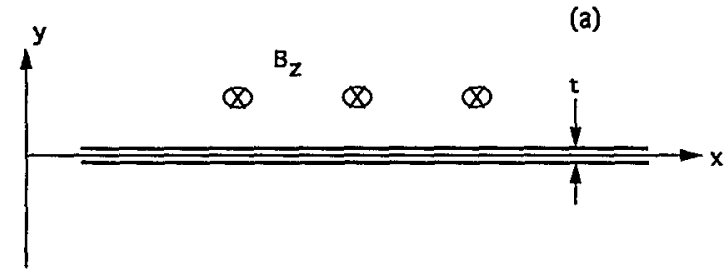

(b)

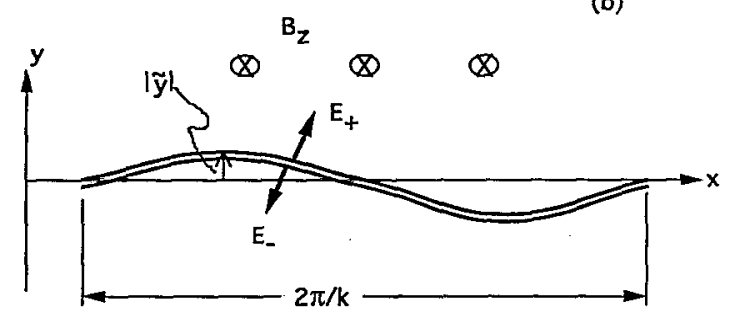

FIG. 2. Schematic (used for stability analysis) of a sheet electron beam focused by a uniform solenoidal magnetic field: (a) unperturbed state, (b) perturbed configuration.

where $\Omega_{0}=\left|q B_{0} / m\right|$. By concentrating on electrons at the beam center, all quantities in the unperturbed state [Fig. $2(a)]$ are approximately zero. For harmonic perturbations of the sort $e^{i(k x-\omega t)}$ one has

$$
\begin{aligned}
& -\omega^{2} \tilde{x}=\left(q \widetilde{E}_{x} / m\right)+i \omega \Omega_{0} \tilde{y}, \\
& -\omega^{2} \tilde{y}=\left(q \widetilde{E}_{y} / m\right)-i \omega \Omega_{0} \tilde{x} .
\end{aligned}
$$

Several approximations are now employed. First, it is assumed that in the perturbed state [Fig. 2(b)], electrons in the beam center experience an electric field $\widetilde{E}$ that is approximately the average of the field at the top $\widetilde{E}_{+}$and bottom $\widetilde{E}_{-}$of the beam,

$$
\widetilde{E} \approx \frac{1}{2}\left(\widetilde{E}_{+}+\widetilde{E}_{-}\right) \text {. }
$$

For electrons at the center of the beam, the perturbed equation of continuity is approximately

$$
\tilde{N} \approx-i N k \tilde{x} \text {. }
$$

Assuming $k t \ll k|\widetilde{y}| \ll 1$ [see Fig. 2(a)], the geometry of Fig. 2(b), continuity of tangential field, and the jump in the normal field components we obtain

$$
\widetilde{E}_{x+}-\widetilde{E}_{x-}=-i\left(N q / \epsilon_{0}\right) k \tilde{y}
$$

and

$$
\widetilde{E}_{y+}-\widetilde{E}_{y-}=\tilde{N} q / \epsilon_{0}
$$

Next, by assuming the time scale of the perturbation to be slow (i.e., $\omega \ll \Omega_{0}$ ), Eqs. (10)-(13) can be combined to yield

$$
\begin{aligned}
& \frac{\widetilde{E}_{y+}+\widetilde{E}_{y-}}{\widetilde{E}_{y+}-E_{y-}}=-\frac{\omega}{k} \frac{2 \epsilon_{0}}{N q} B_{0}, \\
& \frac{\widetilde{E}_{x+}+\widetilde{E}_{x-}}{\widetilde{E}_{x+}-E_{x-}}=+\frac{\omega}{k} \frac{2 \epsilon_{0}}{N q} B_{0} .
\end{aligned}
$$

Boundary conditions at the beam-vacuum edges are obtained by assuming a quasistatic limit (i.e., $k \ll \omega / 2 \pi c$ ) such that fields in the vacuum region are separable in their dependence on $x$ and $y$. For a curl-free electric field, this results in the approximation

$$
\widetilde{E}_{x_{ \pm}} \approx \tau i \widetilde{E}_{y_{ \pm}} \text {. }
$$

Finally, we can combine Eqs. (14) and (15) to obtain

$$
\omega / k= \pm i\left(N q / 2 \epsilon_{0} B_{0}\right),
$$

i.e., a purely growing mode.

We now apply the above model to the case of a sheet beam in a periodic magnetic focusing field. Again, we assume a perturbation of the sort $e^{i(k x-\omega t)}$ as depicted in Fig. 2(b) and assume that the perturbation evolves on a time scale sufficiently long to justify averaging the dynamics over a magnet period [see Eqs. (7)]. Hence, the equations of motion are

$$
-\omega^{2} \tilde{x}=\left(q \widetilde{E}_{x} / m\right)-\left(\Omega_{0}^{2} / 2\right) C_{x} \tilde{x}
$$

and

$$
-\omega^{2} \widetilde{y}=\left(q \widetilde{E}_{y} / m\right)-\left(\Omega_{0}^{2} / 2\right) \tilde{y} .
$$

Using Eqs. (11)-(13) and (15) we obtain for this case the two equations:

$$
\omega^{2}=\left(N q^{2} / 2 m \epsilon_{0}\right) k+\left(\Omega_{0}^{2} / 2\right) C_{x}
$$

and

$$
\omega^{2}=\left(-N q^{2} / 2 m \epsilon_{0}\right) k+\left(\Omega_{0}^{2} / 2\right) .
$$

Normal mode solutions exist only for simultaneous solution of Eqs. (18a) and (18b). Upon substitution this yields

$$
\omega^{2}=\left(\Omega_{0}^{2} / 4\right)\left(1+C_{x}\right) \text {, }
$$

i.e., bounded, harmonic, betatron motion (no growing instability). The validity of this calculation pertains to perturbations for which $\omega / u_{0} \ll k_{m}$, or

$$
\Omega_{0}^{2}\left(1+C_{x}\right) \ll 4 k_{m}^{2} u_{0}^{2},
$$

where $u_{0}$ is the beam's axial velocity.

In conclusion, whereas a sheet electron beam in a uniform solenoidal $1^{3-6,8,9}$ or a slowly varying periodic ${ }^{17}$ magnetic field is unstable to diocotron modes, a sheet beam in a short-period periodic magnetic field should be stable against low-frequency space-charge perturbations. The latter statement is predicated upon the assumption that the periodic magnetic fields are sufficiently strong to permit an equilibrium solution-i.e., that the beam is focused in the bulk sense. Evaluating conditions necessary for such focusing forms the content of the following sections.

Still unresolved are questions associated with dynamics occuring on fast time scales, such as emittance growth and electromagnetic (FFL) instabilities. To rigorously investigate the former requires a $3 \mathrm{D}$ simulation. However, the general qualitative behavior is expected to follow that reported for cylindrical configurations, e.g., emittance growth can occur as the result of beam mismatch ${ }^{21}$ or magnetic-field errors. ${ }^{22}$ Hence, optimizing beam entrance conditions ${ }^{16}$ and minimizing field errors should keep emittance growth low enough for microwave tube applications. Evaluation of emittance growth of periodically focused 


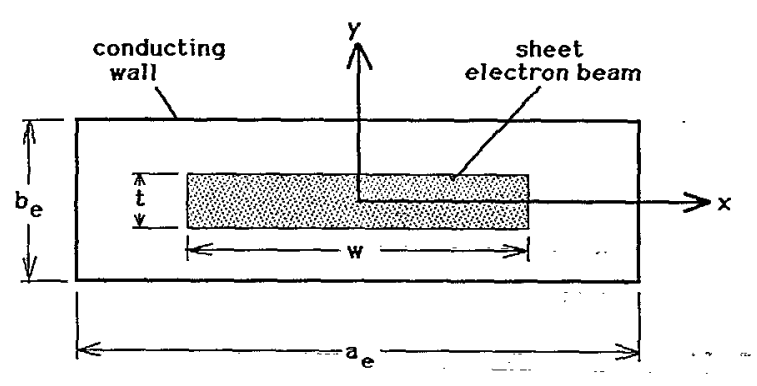

FIG. 3. Cross-sectional view of sheet beam cnclosed in a conducting rectangular waveguide showing relevant beam and waveguide wall dimensions.

sheet beams for more stringent accelerator applications will require more detailed numerical analyses.

Susceptibility to unintended FEL-like electromagnetic instabilities is also theoretically possible, duc to the "wiggling" motion of electrons off the geometric axis as induced by the periodic PCM field. However, whereas the quiver energy scales as (nonrelativistically) $v_{x, \text { rms }}^{2} \approx \Omega_{0}^{2} / 2 k_{m}^{2}$ for near-axis electrons with wiggler focusing, ${ }^{23}$ we have $v_{x, \text { rms }}^{2} \approx \Omega_{0}^{2} y^{2} / 2$ for PCM focusing. Hence,

$$
\left(v_{x, \mathrm{rms}}^{2}\right)_{\mathrm{PCM}} /\left(v_{x, \text { rms }}^{2}\right)_{\text {wiggler }} \approx k_{m}^{2} y^{2},
$$

which will generally be a small number, implying weak growth for FEL-type instabilities.

\section{MAGNETIC AND SPACE-CHARGE ELECTRIC FIELDS FOR A SHEET ELECTRON BEAM IN RECTANGULAR-CROSS-SECTION PCM FOCUSING}

Before evaluating conditions necessary for focusing, we will derive limiting-form expressions for the space-charge electric and magnetic fields associated with sheet electron beams in rectangular-cross-section PCM focusing.

\section{A. Space-charge electrostatic fields of a rectangular sheet electron beam with uniform density and sharp edges}

We seek the space-charge fields for a configuration such as that illustrated in Fig. 3, with beam thickness $t$, beam width $w$, and waveguide cross-section dimensions $a_{e}$ and $b_{e}$. It is assumed that the solution is independent of the waveguide axis dimension $z$. Hence, we seek the solution for electrostatic scalar potential $\Phi_{E}$,

$$
\begin{aligned}
& \nabla^{2} \Phi_{E}(x, y, z)=-\frac{\rho(x, y)}{\epsilon_{0}}, \quad|x| \leqslant a_{e} / 2,|y| \leqslant b_{e} / 2, \\
& \Phi_{E}\left(|x|=a_{e} / 2\right)=\Phi_{E}\left(|y|=b_{e} / 2\right)=0 .
\end{aligned}
$$

A Green's function solution to the relevant equation

$$
\begin{aligned}
& \left(\frac{\partial^{2}}{\partial x^{\prime 2}}+\frac{\partial^{2}}{\partial y^{\prime 2}}\right) G\left(x^{\prime}, x ; y^{\prime}, y\right)=-\frac{\delta\left(x^{\prime}-x\right) \delta\left(y^{\prime}-y\right)}{\epsilon_{0}}, \\
& G\left(\left|x^{\prime}\right|=a_{e} / 2\right)=G\left(\left|y^{\prime}\right|=b_{e} / 2\right)=0,
\end{aligned}
$$

is obtained as

$$
\begin{aligned}
G\left(x^{\prime}, x ; y^{\prime}, y\right)= & \sum_{j=0}^{\infty} g_{j} \cos \left(k_{j} y^{\prime}\right) \cos \left(k_{j} y\right) \\
& \times \sinh \left[k_{j}\left(\frac{a_{e}}{2} \pm x\right)\right] \sinh \left[k_{j}\left(\frac{a_{e}}{2} \mp x^{\prime}\right)\right], \\
& x^{\prime} \gtrless x, \\
g_{j} \equiv & \frac{2}{\epsilon_{0} b_{e} k_{j} \sinh \left(k_{j} a_{e}\right)}, \quad k_{j} \equiv \frac{(2 j+1) \pi}{b_{e}} .
\end{aligned}
$$

The solution to Eq. (21) is therefore

$$
\Phi_{E}(x, y)=\iint_{S_{\mathrm{wg}}} d x^{\prime} d y^{\prime} G\left(x^{\prime}, x ; y^{\prime}, y\right) \rho\left(x^{\prime}, y^{\prime}\right),
$$

where $S_{\mathrm{wg}}$ represents the waveguide cross section. Since the geometry is symmetric about the $y$ axis, we concentrate our computation of $\Phi_{E}$ to the region $x \geqslant 0$. Taking the case of a uniform distribution of beam density $n$ with "sharp" edges

$$
\rho(x, y) \equiv\left\{\begin{array}{l}
-e n, \quad|y| \leqslant t / 2,|x| \leqslant w / 2 \\
0, \quad \text { otherwise }
\end{array}\right.
$$

(where $n$ is a constant) one obtains from Eqs. (23) and (24)

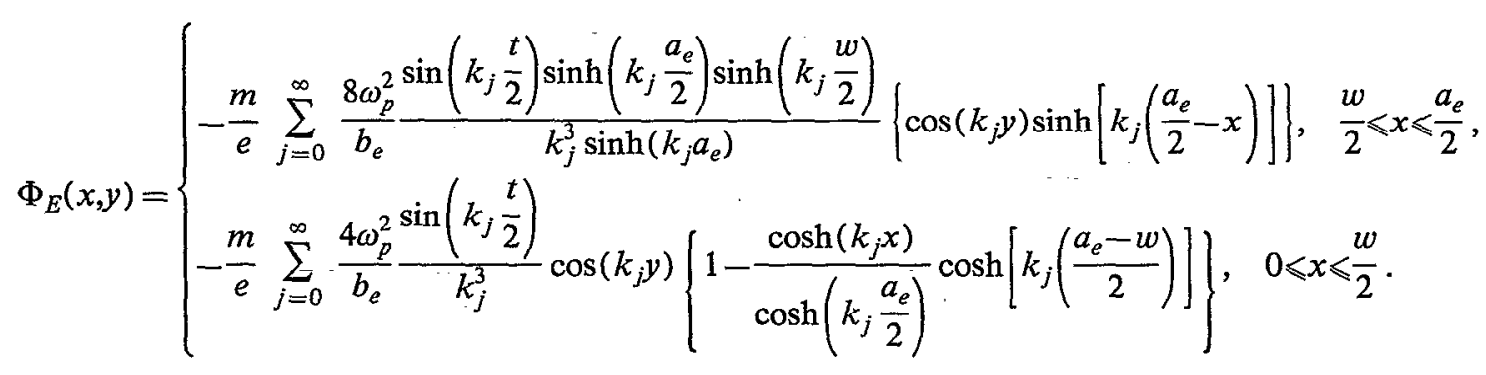

It is useful to obtain the form of this expression in the limit that we can ignore the sidewalls at $x= \pm a_{e} / 2$. Evaluating Eq. (26) in the limit $a_{e} \rightarrow \infty$ yields 


$$
\Phi_{E}(x, y)=-\frac{m}{e} \sum_{j=0}^{\infty} \frac{4 \omega_{p}^{2}}{b_{e}} \frac{\cos \left(k_{j} y\right) \sin \left[k_{j}(t / 2)\right]}{k_{j}^{3}}\left(1-\frac{e^{-k_{j}[(w / 2)+x]}+e^{-k_{j}[(w / 2)-x]}}{2}\right),
$$

for $|x| \leqslant w / 2$. This expression becomes accurate when $k_{j}\left(a_{e}-w\right) / 2 \geqslant 2$, or whenever $\left(a_{e}-w\right) \gtrsim 1.3 b_{e^{e}}$. In this limit it is identical to that which one obtains for the sheet beam positioned between two infinite parallel planes as shown in Fig. 4 and associated with the Green's function

$$
G_{\|}\left(x, x^{\prime} ; y, y^{\prime}\right)=\sum_{j=0}^{\infty} \frac{2 \cos \left(k_{j} y^{\prime}\right) \cos \left(k_{j} y\right)}{\epsilon_{0} b k_{j}} \frac{e^{ \pm k_{j}\left(x-x^{\prime}\right)}}{2}, x \lessgtr x^{\prime} .
$$

For stability and focusing calculations, expressions for the electric field, $\mathbf{E}=-\nabla \Phi_{E}$ will be preferred. As a check on the validity of our expressions above, it is instructive to first consider the electric field for an infinitely wide sheet beam of thickness $t / 2$, placed between two conducting parallel plates that are separated by a spacing of $b_{e}$. This can be rather trivially obtained by direct integration of Gauss's law to obtain

$$
\mathbf{E}=\left\{\begin{array}{l}
(-m / e) \omega_{p}^{2} y \hat{\mathbf{y}}, \quad 0 \leqslant y \leqslant t / 2, \\
(-m / e) \omega_{p}^{2}(t / 2) \hat{\mathbf{y}}, \quad t / 2 \leqslant y \leqslant b_{e} / 2
\end{array}\right.
$$

Alternatively, one should obtain the same result by taking the negative gradient of Eq. (27) in the limit $w / 2 \rightarrow \infty$ to obtain

$$
E_{y}(y) \stackrel{\substack{\text { infinitely wide beam } \\ w / 2 \rightarrow \infty}}{\longrightarrow}-\frac{m}{e} \sum_{j=0}^{-\infty} \frac{4 \omega_{p}^{2}}{b_{e}} \frac{\sin \left[k_{j}(t / 2)\right]}{k_{j}^{2}} \sin \left(k_{j} y\right)
$$

It has been verified that Eqs. (29) and (30) are in exact agreement by completing the sum numerically.

More generally, the electric field for a sheet beam with uniform density and "sharp" edges can be obtained by taking the negative gradient of Eq. (26) or Eq. (27), depending on which limiting case is of interest. As an illustration, we compute the electric-field components for the case of a wide sheet beam with uniform density and sharp (abrupt) edges at $|x|=w / 2$ where the effect of the side walls can be neglected [e.g., using Eq. (27)]:

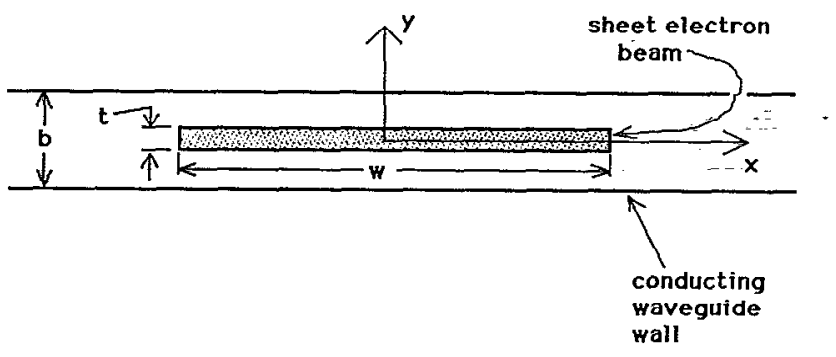

FIG. 4. Rectangular sheet beam confined between two conducting parallel planes.

$$
\begin{aligned}
E_{x}(x, y)= & -\frac{m}{e}-\sum_{j=0}^{\infty} \frac{2 \omega_{p}^{2}}{b_{e}} \frac{\sin \left[k_{j}(t / 2)\right]}{k_{j}^{2}} \cos \left(k_{j} y\right) \\
& \times \exp \left(-k_{j} \frac{w}{2}\right) \sinh \left(k_{j} x\right), \\
E_{y}(x, y)= & -\frac{m}{e} \sum_{j=0}^{\infty} \cdot \frac{4 \omega_{p}^{2}}{b_{e}} \frac{\sin \left[k_{j}(t / 2)\right]}{k_{j}^{2}} \sin \left(k_{j} y\right) \\
= & \times\left(1-\frac{e^{-k_{j}(w / 2+x)}+e^{-k_{j}(w / 2-x)}}{2}\right) .
\end{aligned}
$$

\section{B. Space-charge electrostatic fields of a rectangular sheet electron beam with nonuniform density or diffuse edges}

Equations (31) provide expressions for the electricfield components of a wide rectangular sheet beam with uniform density, abrupt edges at $|\boldsymbol{x}|=w / 2$, and confined between two infinitely wide parallel conducting plates. The assumptions" of "uniform density and abrupt edges at $|x|=w / 2$ may not always be applicable or desirable and in this sub-section we investigate formulas for the electrostatic space-charge field when the beam has diffuse edges that are nominally located at $|x|=w / 2$. We will start with a very simplificd model and formula, followed by a more exact derivation to establish validity regimes for the simpler expression.

First, we consider a simplified model in which the $x$ and $y$ dependencies are approximately separable. In particular, we consider Poisson's equation for a wide rectangular sheet electron beam confined between parallel conducting plates with beam density $n(x)$ that is uniform in $y$ but variable in $x$ (for example, near the beam edge):

$$
\frac{\partial^{2} \Phi_{E}}{\partial x^{2}}+\frac{\partial^{2} \Phi_{E}}{\partial y^{2}}=\frac{e}{\epsilon_{0}} n(x)
$$

When the density varies sufficiently slowly in $x$ (criteria to bc determined later), we can expect

$$
\frac{\partial^{2} \Phi_{E}}{\partial x^{2}} \ll \frac{\partial^{2} \Phi_{E}}{\partial y^{2}}
$$

or

$$
\frac{\partial^{2} \Phi_{E}}{\partial y^{2}} \approx \frac{e}{\epsilon_{0}} n(x)
$$




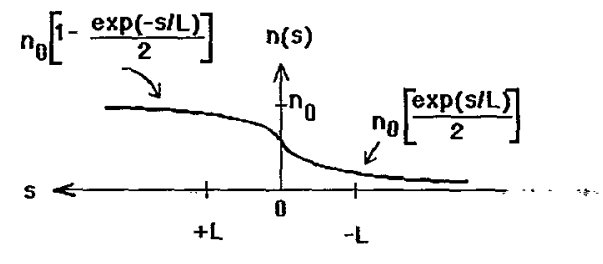

FIG. 5. Horizontal density profile used to compare approximate and exact space-charge-ficld solutions for a beam with diffuse cdges [Egs. (33)].

For a beam of thickness $t$ and symmetrically confined between conducting planes separated by a distance $b_{e}$, one readily obtains a solution for the potential

$\Phi_{E}(x, y)= \begin{cases}\frac{e n(x)}{2 \epsilon_{0}}\left[y^{2}-t\left(\frac{b_{e}}{2}-\frac{t}{4}\right)\right], & |y| \leqslant \frac{t}{2}, \\ \frac{e n(x)}{2 \epsilon_{0}} t\left(y-\frac{b_{e}}{2}\right), & \frac{t}{2} \leqslant|y| \leqslant \frac{b_{e}}{2},\end{cases}$

and for the electric-field components in the region $|y| \leqslant t / 2$,

$$
\begin{aligned}
& E_{x}(x, y) \approx-\frac{e}{2 \epsilon_{0}}\left[y^{2}-t\left(\frac{b_{e}}{2}-\frac{t}{4}\right)\right] \frac{\partial n(x)}{\partial x}, \\
& E_{y}(x, y) \approx-\left(e / \epsilon_{0}\right) n(x) y .
\end{aligned}
$$

To establish validity restrictions on Eqs. (35) for the case of a sheet beam symmetrically placed between conducting planes, we again perform a Green's function solution for the electrostatic fields. In this case, however, we consider a beam with a prescribed diffuse density variation. It will be convenient to measure horizontal displacement from the beam edge by introducing the position variable $s \equiv w / 2-x$. To keep the analysis clear and tractable, we select a particular representative density variation near the nominal beam edge at $s=0$ :

$$
n(x) \rightarrow n(s)=\left\{\begin{array}{l}
\left(n_{0} / 2\right) \exp (s / L), \quad s \leqslant 0, \\
n_{0}\left[1-\frac{1}{2} \exp (-s / L)\right], \quad s \geqslant 0,
\end{array}\right.
$$

schematically illustrated in Fig. 5. The horizontal electricfield component can be calculated as

$$
\begin{aligned}
E_{s}(s, y) & =-\frac{\partial \Phi_{E}}{\partial s} \\
& =\int_{-\infty}^{\infty} d s^{\prime} e n\left(s^{\prime}\right) \int_{-t / 2}^{+t / 2} d y^{\prime} \frac{\partial G_{\|}\left(s, s^{\prime} ; y, y^{\prime}\right)}{\partial s}
\end{aligned}
$$

where $G_{\|}\left(s, s^{\prime} ; y, y^{\prime}\right)$ is obtained from Eq. (28). The exact result for this case is

$$
\begin{aligned}
E_{s}(s, y)= & \frac{e n_{0}}{\epsilon_{0}} \sum_{j=0}^{\infty} \frac{2 \sin \left[k_{j}(t / 2)\right]}{b_{e} k_{j}^{2}} \cos \left(k_{j} y\right) \\
& \times\left(\frac{k_{j} L \exp (-s / L)-\exp \left(k_{j} s\right)}{k_{j}^{2} L^{2}-1}\right),
\end{aligned}
$$

where $k_{j}$ has the same definition as before. Introducing the normalized quantities $\hat{L}=L / b_{e}, \hat{s}=s / b_{e}, \hat{t}=t / b_{e}, \hat{y}=y / b_{e}$, and $\hat{E}=E_{s} / E_{0}$, where $E_{0}=-e n_{0} b_{e} / \epsilon_{0}$, Eq. (38) can be rewritten as

$$
\begin{aligned}
\hat{E}_{s}(s, \hat{y}) & \\
= & -\sum_{j=0}^{\infty} \frac{2 \sin \left(\frac{(2 j+1) \pi \hat{t}}{2}\right)}{(2 j+1)^{2} \pi^{2} \hat{t}} \cos [(2 j+1) \pi \hat{y}] \\
& \times\left(\frac{(2 j+1) \pi \hat{L} \exp \left(-\frac{\hat{s}}{\hat{L}}\right)-\exp [-(2 j+1) \pi \hat{s}]}{(2 j+1)^{2} \pi^{2} \hat{L}^{2}-1}\right) .
\end{aligned}
$$

We now proceed to compare the exact expression (39a) with the approximate expression (35a) for the case of the density variation specified in Eq. (36). In terms of the normalized quantities, Eq. (35a) using Eq. (36) is rewritten as

$$
\hat{E}_{s}(s, y) \approx-\frac{1}{4 \hat{L}}\left[\frac{\hat{y}^{2}}{\hat{t}}-\left(\frac{1}{2}-\frac{\hat{t}}{4}\right)\right] \exp \left(-\frac{\hat{s}}{\hat{L}}\right) .
$$

The exact expression (39a) is then computed by numerically summing over a large but finite number of terms. This summation is facilitated by noting that for $(2 j+1) \pi \hat{t} /$ $2<1, E_{s} \sim(2 j+1)^{-1}$, whereas for $(2 j+1) \pi \hat{t} / 2>1$,

$$
\hat{E}_{s} \sim \frac{\sin [(2 j+1) \pi(\hat{t} / 2)]}{(2 j+1)^{3} \pi^{3} t^{2} \hat{L}} \sim \frac{1}{(2 j+1)^{3}},
$$

a rapidly converging series. Consequently, the sum in Eq. (39a) can be well approximated by truncation after $j=1 /$ $\pi \hat{t}-\frac{1}{2}$. Equations (39a) and (39b) have been plotted in Figs. $6($ a) and $6(\mathrm{~b})$ for $\hat{L}=3$ and $\hat{L}=1$, respectively. From the two plots, it can be inferred that the simplified expressions [Eqs. (35) or (39b)] can be used with reasonable accuracy (within $10 \%$ ) whenever $\hat{L}=L / b_{e} \geqslant 1$. Furthermore, the simplified expressions generally err in a conservative manner (higher $E$-field estimate than the exact formula).

In summary, Eqs. (31) and (35) provide simplified limiting forms for the electric space-charge fields of rectangular-cross-section sheet beams for the cases of uniform density with sharp edges [Eqs. (31)] and sheet beams with density gradients along the horizontal dimension [Eqs. (35)]. With reference to Fig. 3, both equations are quantitatively valid for $\left(a_{e}-w\right) \gtrsim 1.3 b_{e}$. Equations (35) are generally applicable to density gradients at the beam edge or in the beam center, provided the density gradient scale length is not too abrupt, i.e., $L / b_{e} \geqslant 1$.

\section{Magnetostatic fields for 2D PCM focusing}

In this subsection, we investigate periodically cusped magnetic-field configurations that will provide sheet beam focusing in both transverse planes. At first glance, one may be tempted to consider either curved-pole ${ }^{24}$ or cantedpole $^{25}$ configurations which have been successfully employed with wiggler focusing. However, in periodic-cusped focusing, midplane $(y=0)$ electrons experience no trans- 

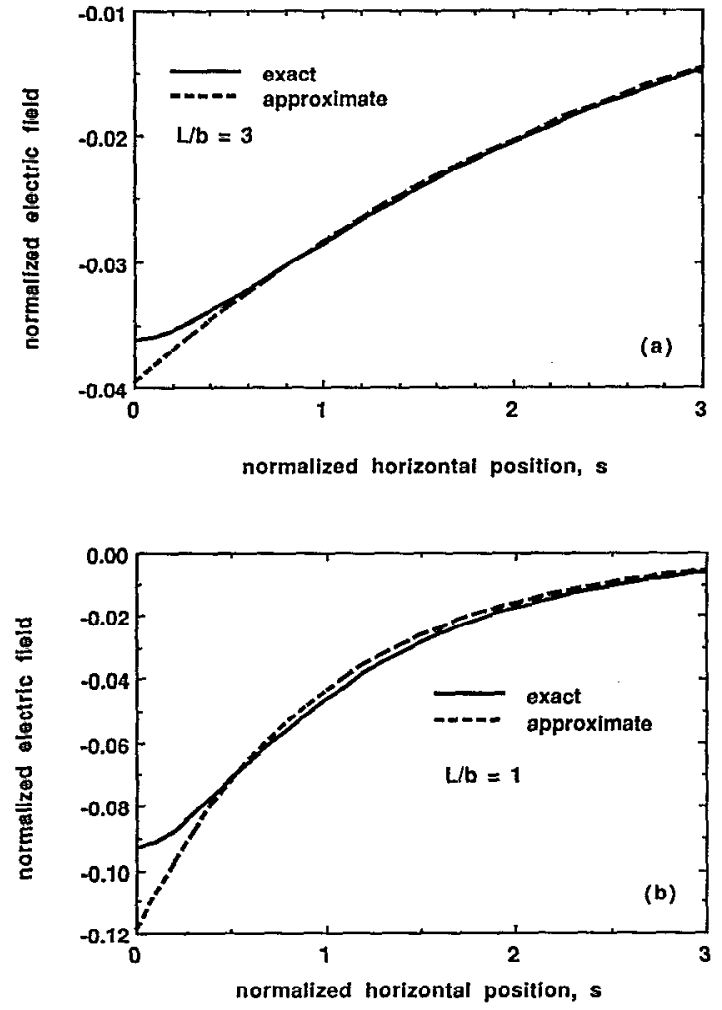

FIG. 6. Comparison of exact [Eq. (33a)] and approximate [Eq. (33b)] space-charge-field solutions for a beam with diffuse edges. $s$ is a horizontal position coordinate measuring displacement relative to the beam edge (at $s=0$ ) and normalized to the waveguide wall separation distance $b_{e}$. (a) $t / b_{e}=0.1$ and $L / b_{e}=3$; (b) $t / b_{e}=0.1$ and $L / b_{e}=1$.

verse force or quiver velocity. Hence, neither the curvedpole nor the canted-pole configuration will provide horizontal focusing for midplane electrons-which generally represent a large fraction of the sheet beam. Instead, we will consider two alternative configurations.

The first configuration-henceforth referred to as a "closed" configuration-is that displayed in Fig. 1. This structure is topologically equivalent to PPM focusing of round beams and can be envisioned as the result of compressing a circular-cross-section PPM structure in one transverse $(y)$ dimension while stretching the configuration in the other transverse dimension $(x)$. Focusing occurs in the narrow dimension by the simultaneous existence of $B_{y}$ and $B_{z}$ components, while focusing in the wide transverse dimension results from the presence of $B_{x}$ and $B_{z}$ components.

In principle, the fields for the closed configurationof Fig. 1 can be obtained from a scalar magnetic potential $\Phi_{M}$ that satisfies Laplace's equation along with appropriate boundary conditions at the inside edges of the magnet,

$$
\nabla^{2} \Phi_{M}(x, y, z)=0, \quad|x| \leqslant a_{m} / 2, \quad|y| \leqslant b_{m} / 2 .
$$

In the limit of an infinitely wide problem (i.e., $a_{m} \rightarrow \infty$ ), the problem reduces to two dimensions ( $y$ and $z$ ) and can be solved by separationof variables in a manner similar to that of Refs. 26 and 27 for wiggler magnets. For finite $a_{m}$, however, the problem is no longer separable. Nevertheless,

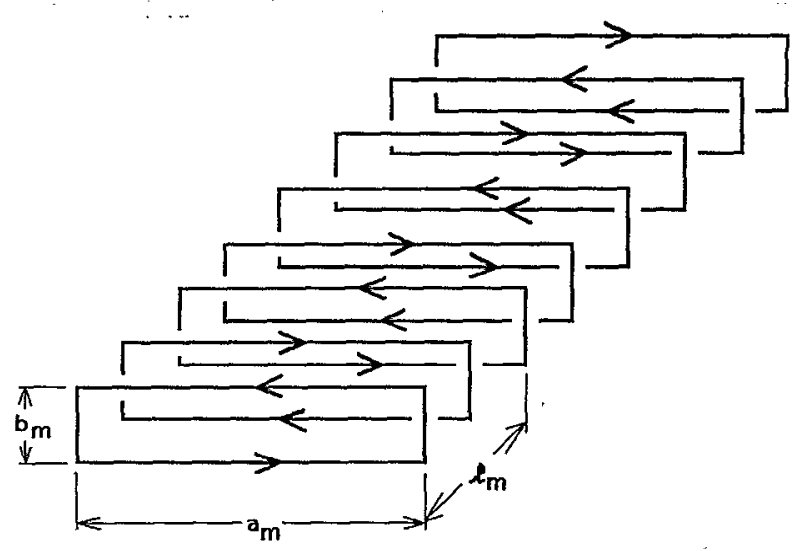

FIG. 7. Filamentary model (without iron pole pieces) of rectangular PCM configuration.

in the magnet gap region far from the magnet edges one intuitively expects approximately separable solutions (from Sec. II):

$$
\begin{aligned}
& B_{x} \approx-B_{0}\left(k_{x} / k_{m}\right) \sinh \left(k_{x} x\right) \cosh \left(k_{y} y\right) \cos \left(k_{m} z\right), \\
& B_{y} \approx-B_{0}\left(k_{y} / k_{m}\right) \cosh \left(k_{x} x\right) \sinh \left(k_{y} y\right) \cos \left(k_{m} z\right), \\
& B_{z} \approx B_{0} \cosh \left(k_{x} x\right) \cosh \left(k_{y} y\right) \sin \left(k_{m} z\right),
\end{aligned}
$$

where, to satisfy Laplace's equation as well as to obtain $B_{y}\left(b_{m} / 2\right)=B_{x}\left(a_{m} / 2\right)$, we take

$$
\begin{aligned}
& k_{x} \approx \frac{k_{m}}{\sqrt{1+a_{m}^{2} / b_{m}^{2}}}, \\
& k_{y} \approx \frac{k_{m}}{\sqrt{1+b_{m}^{2} / a_{m}^{2}}} .
\end{aligned}
$$

To support this approximation, we have used the BiotSavart formula and numerically calculated the fields for the filamentary closed PCM configuration illustrated Fig. 7. The transverse field variation of Fig. 7 is expected to be similar to that of Fig. 1, with the principal exception that higher-harmonic content should be observed for the configuration of Fig. 1 due to the presence of the lowreluctance iron pole pieces. Specific results for the $z$ component of the case $a_{m}=5.0, b_{m}=0.5, k_{m}=2 \pi$ (i.e., all dimensions are normalized to the magnet period $l_{m}$ ), are plotted in Fig. 8. Generally, for $|y| \leqslant 0.1$ and $|x| \leqslant 1.0$ it was observed that the above approximate expressions [Eqs. (2) and (41)] provide a very good fit to the computed field values. More exact numerical simulations including the effects of ferritic pole pieces are planned for the future. For the purposes of this article, however, we will make use of the approximate expressions given above.

A second configuration is also considered, namely the "open" structure of Fig. 9 with offset pole pieces. This offset-pole-piece configuration has already been successfully used for two-plane wiggler focusing of low-spacecharge, relativistic sheet electron beams. ${ }^{15}$ The two-plane focusing properties of this arrangement can be appreciated from inspection of its magnetic equivalent in Fig. 10. Fo- 

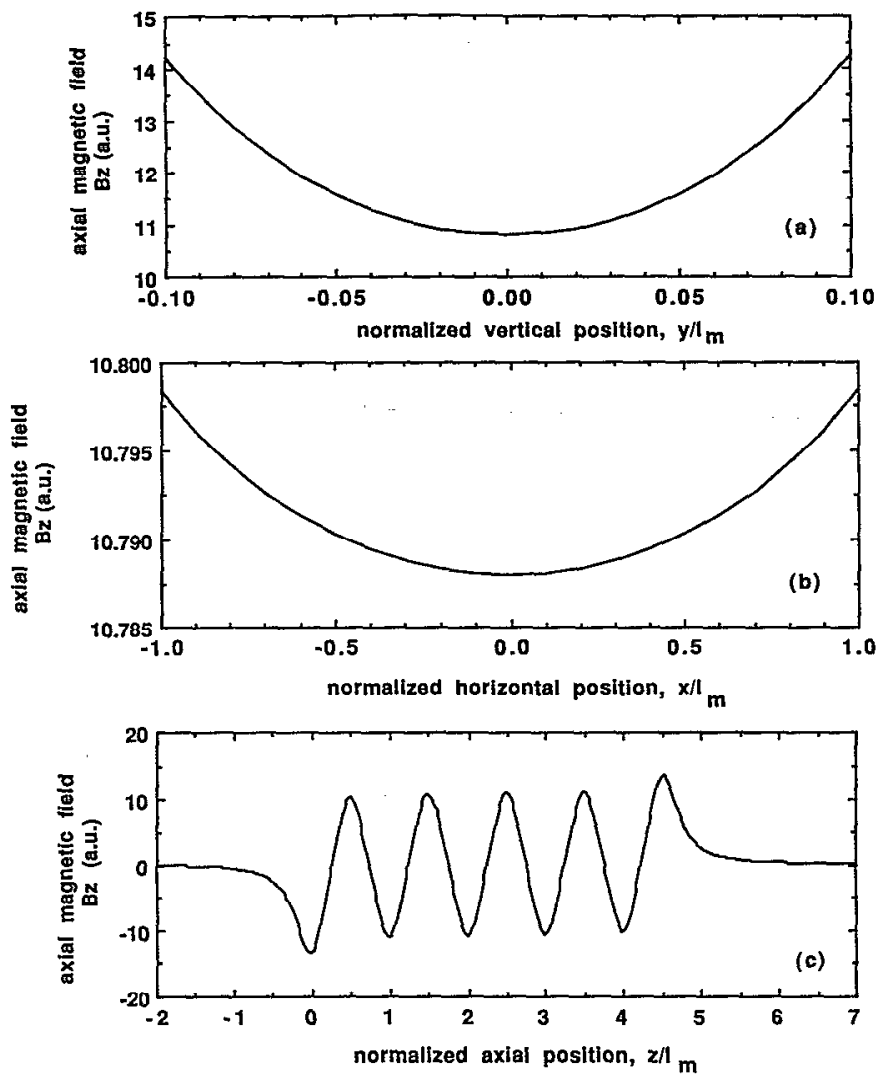

FIG. 8. Numerically calculated axial magnetic-field component $B_{z}$ of the filamentary PCM configuration displayed in Fig. 7 [10 coils placed at $z=(0,0.5,1.0, \ldots, 4.0,4.5)]$. (a) $B_{z}$ vs $x$ at $y=0$ and $z=2.5$, (b) $B_{z}$ vs $y$ at $x=0$ and $z=2.5$, and (c) $B_{z}$ vs $z$ for $x=0$ and $y=0$.

cusing in the narrow $(y)$ dimension works on the ponderomotive (periodic fields) principle, whereas focusing at the beam edges in the wide transverse dimension results from the zeroth-order $v_{z} B_{y}$ forces.

To model the fields of the configuration in Fig. 9 (or Fig. 10) we will superpose periodic fields for an "infinitely wide" PCM structure with fields resulting from four semiinfinite sheets of magnetic "surface charge", as depicted in

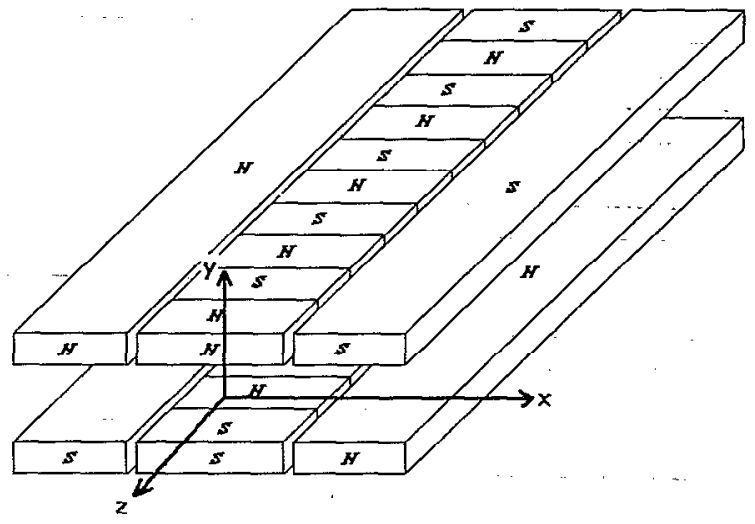

FIG. 10. An open-sided PCM configuration equivalent to the offset pole piece configuration of Fig. 9. Again, magetic polarity labels $(N, S)$ indicate the field polarity on the inside or gap-side faces of the magnet poles.

Fig. 11. With this approach, we can solve for the periodic field, $\mathbf{B}_{\mathrm{pcm}}$, using Eq. (40) and separation of variables. Following the derivations of Refs. 26 and 27, and keeping just the lowest-order harmonic terms, the result is

$$
B_{y, \mathrm{pcm}} \approx-B_{0} \sinh \left(k_{m} y\right) \cos \left(k_{m} z\right)
$$

and

$$
B_{z, \mathrm{pcm}} \approx B_{0} \cosh \left(k_{m} y\right) \sin \left(k_{m} z\right) .
$$

The side or "offset" fields $\mathbf{B}_{\text {off }}$ can be obtained from Coulomb's law-i.e., by integrating over the "surface magnetic charge" sources,

$$
\begin{aligned}
\mathbf{B}_{\text {off }}= & C_{B} \int_{\text {sources }}\left(\frac{\left(x-x^{\prime}\right) \rho_{m s} d x^{\prime}}{\left[\left(x-x^{\prime}\right)^{2}+\left(y-y^{\prime}\right)^{2}\right]} \hat{\mathbf{x}}\right. \\
& \left.+\frac{\left(y-y^{\prime}\right) \rho_{m s} d x^{\prime}}{\left[\left(x-x^{\prime}\right)^{2}+\left(y-y^{\prime}\right)^{2}\right]} \hat{\mathbf{y}}\right),
\end{aligned}
$$

where $C_{B}$ is a dimensionalizing constant. Equation (43) has been solved for the semi-infinite magnetic charge

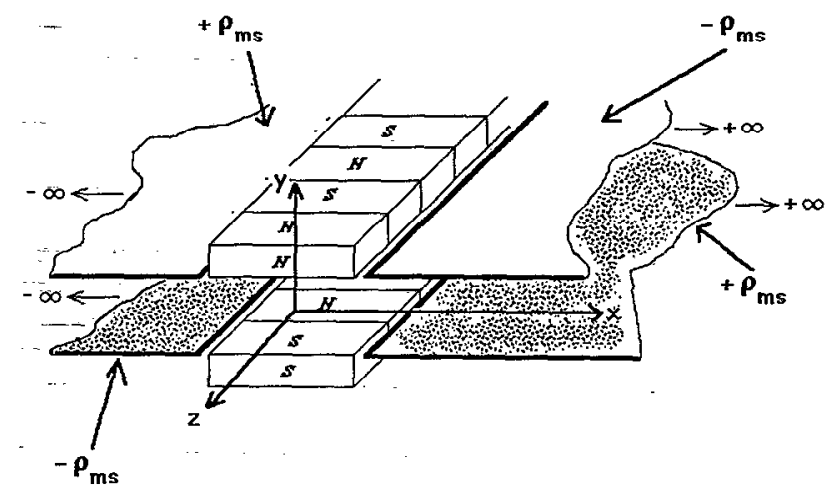

FIG. 11. Magnetic equivalent of open-sided configurations in Figs. 9 and 10 achieved by replacing offset pole regions with semi-infinite sheets of magnetic "surface charge."
FIG. 9. Open-sided PCM configuration using offset pole pieces. Magnetic polarity labels $(N, S)$ indicate the field polarity on the inside or gap-side faces of the magnet poles. 


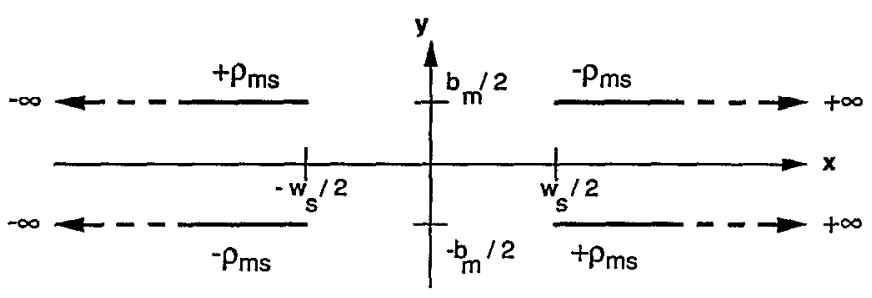

FIG. 12. Two-dimensional view of the semi-infinite magnetic surface charge sheets of Fig. 11, indicating and labeling their relative positions.

source distribution pictured in Fig. 12, subject to the condition that $\mathbf{B}_{\text {off } \rightarrow \pm} \pm B_{s} \hat{y}$ at $x \rightarrow \pm \infty$. In particular, the $y$ component of $\mathbf{B}_{\text {off }}$ is

$$
\begin{aligned}
B_{y, \text { off }}= & \frac{B_{s}}{\pi}\left[\arctan \left(\frac{w_{s} / 2+x}{\left|y-b_{m} / 2\right|}\right)\right. \\
& \left.-\arctan \left(\frac{w_{s} / 2-x}{\left|y-b_{m} / 2\right|}\right)\right] .
\end{aligned}
$$

The fields of the open-side configuration of Figs. 9 or 10 are therefore modeled as the superposition $\mathbf{B}=\mathbf{B}_{\mathrm{pcm}}+\mathbf{B}_{\text {off }}$ using Eqs. (42) and (44).

\section{FOCUSING AND BEAM MATCHING}

In this section, we review conditions for bulk beam focusing and beam matching. In general, the problem of matching a rectangular sheet beam with two-planefocusing periodic magnetic fields is a nonlinear, nonseparable problem involving the balance of magnetic focusing forces, space-charge defocusing forces, and transverse kinetic pressure associated with finite beam emittance. Future numerical studies of this problem are planned, but they are beyond the scope of this article. Hence, present discussions will be limited to approximate analyses associated with zero emittance ("cold") laminar beams.

\section{A. Basic focusing in two dimensions}

To clarify the discussion, we first consider general guidelines for PCM focusing of an infinitely wide sheet beam with uniform density. In this limit, the problem depends only on the $y$ dimension, for which the relevant equation of motion is [see Eq. (17b)]

$$
\frac{d v_{y}}{d t} \approx \frac{q}{m} E_{y}-\frac{\Omega_{0}^{2}}{2} y \approx \omega_{p}^{2} y-\frac{\Omega_{0}^{2}}{2} y
$$

Hence, we can expect beam focusing for

$$
\Omega_{0}^{2} \geqslant 2 \omega_{p}^{2}
$$

Furthermore, to ensure the validity of the period-averaged equations, as well as to ensure stability against diocotron modes, we require a spatial magnet period short enough to satisfy $k_{\beta} \ll k_{m}, \quad k_{p}=\omega_{p} / u_{0} \ll k_{m}, \quad$ and $k_{d}=2 \pi / L_{d} \ll k_{m}$, where $k_{\beta}$ is the betatron wave number and $L_{d}$ has been defined in Eq. (1). Simultaneous satisfaction of these constraints yields a general guideline for a stable confinement regime of periodically focused (nonrelativistic) sheet electron beams,

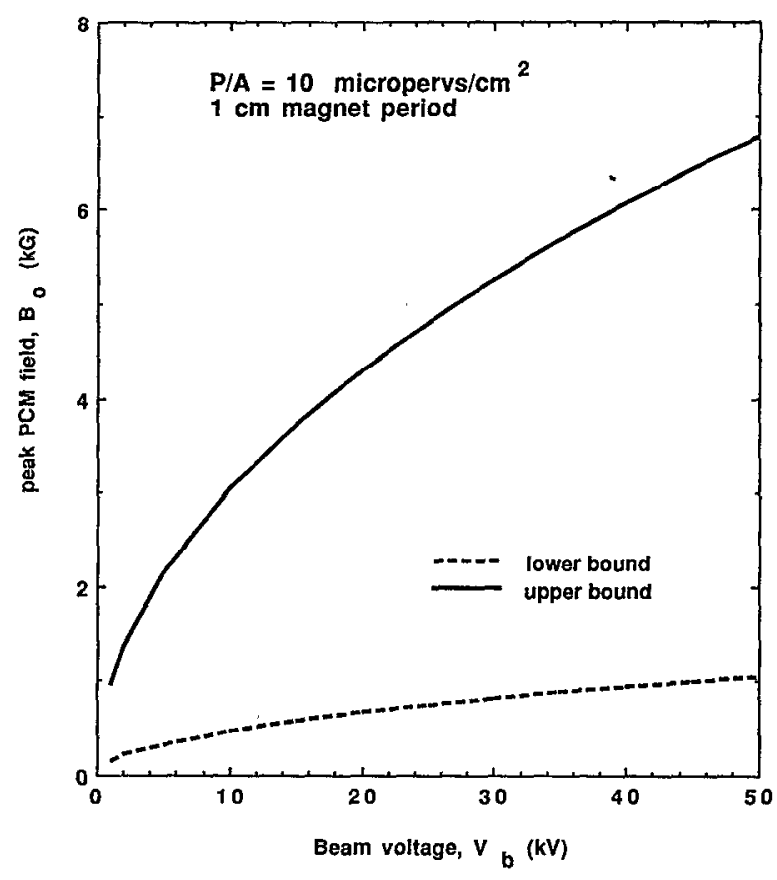

FIG. 13. Stability regime for a PCM focused sheet electron beam with perveance density $P / A=10^{-5}$ pervs $/ \mathrm{cm}^{2}$ and PCM magnet period $l_{m}=1$ cm.

$$
2 \omega_{p}^{2} \leqslant \Omega_{0}^{2} \ll 2 k_{m}^{2} u_{0}^{2} \text {. }
$$

Based on the definitions of the various parameters in Eq. (46), an alternative form is

$$
\left(2.1 \times 10^{-11}\right) n_{0} \leqslant B_{0}^{2} \ll\left(9.2 \times 10^{-4}\right)\left(V_{b} / l_{m}^{2}\right),
$$

where $n_{0}$ is the beam density in $\mathrm{cm}^{-3}, B_{0}$ is the peak PCM field amplitude in $\mathrm{kG}, l_{m}$ is the PCM period in $\mathrm{cm}$, and $V_{b}$ is the beam voltage in volts. Further advantage is gained by noting that

$$
n_{0}=J_{b} / e u_{0} \text {. }
$$

Also, for a thermionic Pierce gun sheet beam source (space-charge limited) the current density is related to the beam voltage as

$$
J_{b}\left(\mathrm{~A} / \mathrm{cm}^{2}\right)=(P / A) V_{b}^{3 / 2},
$$

where $P / A$ represents the perveance per unit area of the beam in units of pervs $/ \mathrm{cm}^{2}$ and $V_{b}$ is in volts. Thus, Eq. (46) can be expressed as

$$
2.2(P / A) V_{b} \leqslant B_{0}^{2} \ll \frac{9.2 \times 10^{-4}}{l_{m}^{2}} V_{b} .
$$

As an illustration, consider the representative case of $P / A=10 \mu$ pervs $/ \mathrm{cm}^{2}$ - a value readily achieved in present commercial Pierce-type thermionic electron guns-and $l_{m}=1 \mathrm{~cm}$. The corresponding upper and lower bounds on values of peak PCM magnetic field for stable sheet beam confinement have been plotted in Fig. 13 for beam voltages ranging between 1 and $50 \mathrm{kV}$. From these values it can be 
assured that exceeding the lower bound while staying significantly less than the upper bound is well within the capability of modern magnet technology.

We next turn to consideration of bulk focusing of sheet beams in the horizontal dimension. For the closed magnetic configuration of Fig. 7 and a sheet beam near $y \approx 0$, we assume the approximate equation of motion [see $\mathrm{Eq}$. $(17 b)]$

$$
\frac{d v_{x}}{d t} \approx \frac{q}{m} E_{x}-\frac{\Omega_{0}^{2}}{2} \frac{k_{x}^{4}}{k_{m}^{4}} x .
$$

For a beam with uniform density and sharp edges, we use Eq. (31a) for the electric field. Looking at the beam edge $(x=w / 2)$ and taking $y \approx 0$, the condition for magnetic focusing to exceed space-charge defocusing is

$$
\omega_{p}^{2} \sum_{j=0}^{\infty} \frac{2 \sin [(2 j+1)(\pi / 2)(t / b)]}{(2 j+1)^{2} \pi^{2}} \leqslant \frac{\Omega_{0}^{2}}{2} \frac{k_{x}^{4}}{k_{m}^{4}} \frac{w}{2 b} .
$$

To illustrate, we consider the particular case of $t / b=0.1$ and $w / b=6$. Furthermore, from the previous discussion of the closed PCM configuration and with reference to Fig. 5, we can take $k_{x}^{4} / k_{m}^{4} \approx b^{4} / a^{4}$ for which we consider the representative case of $b / a=0.1$. For these values, Eq. (48) approximately reduces to

$$
300 \omega_{p}^{2} \leqslant \Omega_{0}^{2}
$$

or

$$
330(P / A) V_{b} \leqslant B_{0}^{2},
$$

which is significantly more restrictive than for focusing in the $y$ dimension.

By comparison, basic focusing in the $x$ direction using the offset-pole or open-side configuration appears much easier. In this case, the equation of motion for electrons at the beam edge is

$$
\frac{d v_{x}}{d t} \approx \frac{q}{m} E_{x}-\frac{q}{m} u_{0} B_{y, \text { off }}
$$

Again, we use Eq. (31a) for the space-charge electric field at the beam edge and $B_{y, \text { off }}$ is based on the model of Eq. (44). For illustration, we set $b_{m}=b_{e}, w_{s}=w, x=w / 2, y=0$, and select representative values of $t / b=0.1$ and $w / b=6$ yielding the horizontal focusing condition

$$
0.4 \omega_{p}^{2} b / u_{0} \leqslant \Omega_{s},
$$

where $\Omega_{s} \equiv q B_{s} / m$. Converting as before to an alternative form, we obtain

$$
130(P / A) b \sqrt{V_{b}} \leqslant B_{s},
$$

where $P / A$ is in pervs $/ \mathrm{cm}^{2}, V_{b}$ is in volts, the waveguide wall separation $b$ is in $\mathrm{cm}$, and $B_{s}$ is in kG. In Fig. 14 we have plotted Eqs. (50) and (52) for the case where $P / A$ $=10 \mu$ pervs $/ \mathrm{cm}^{2}$ and $b=0.5 \mathrm{~cm}$. The fact that $B_{s}$ is considerably less than $B_{0}$ (by several orders of magnitude) illustrates the greater effectiveness of open-side focusing.
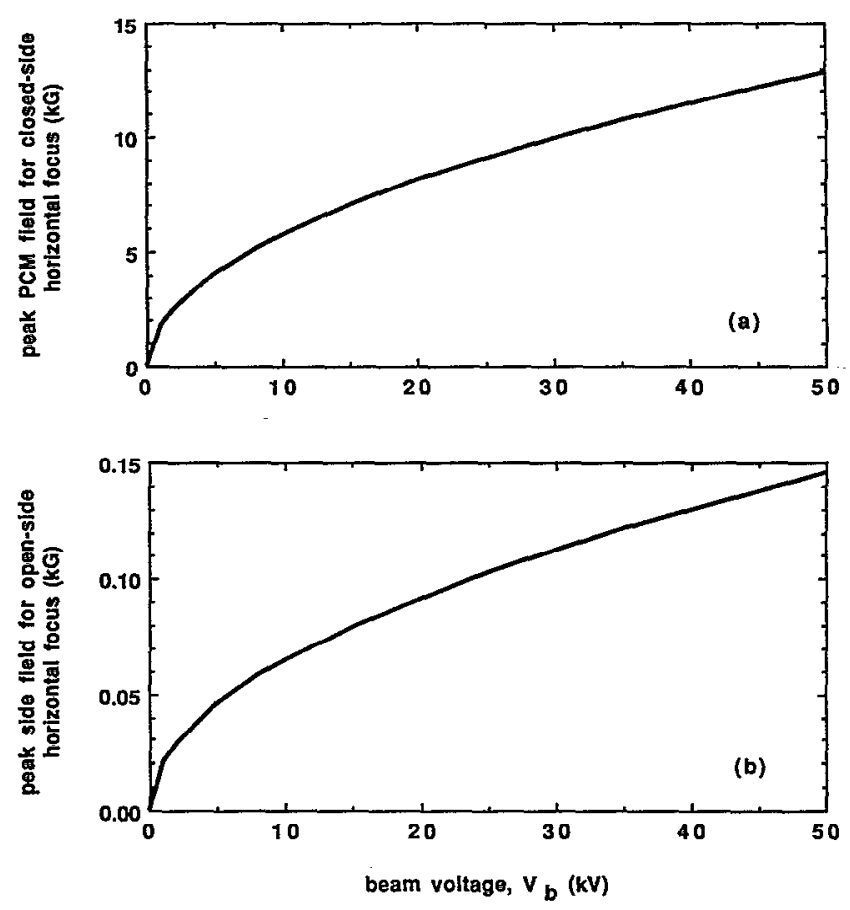

FIG. 14. Comparison of peak magnetic fields needed to horizontally focus a sharp-edged rectangular sheet beam with $t / b=0.1$ and $w / b=6$ : (a) peak PCM field intensity needed for horizontal focusing with closed-side configuration; (b) peak side field intensity needed for horizontal focusing with open-side configuration. Both figures assume a perveance density of $P / A=10^{-5}$ pervs $/ \mathrm{cm}^{2}$. Note the large difference in vertical axis scale between the two figures.

\section{B. Matching with sheet beams having constant thickness}

From the discussion above, stable focusing of sheet electron beams with appreciable space charge appears to be within the reach of present technology; however, most applications of this capability (such as coherent microwave sources or accelerators) are also interested in low-velocity spread or low emittance. From this perspective, the situation is much more complicated. In general, controlling emittance growth in periodically focused beams requires beam matching at injection ${ }^{16,21}$ - that is, a precise balance of space charge, magnetic focusing, and transverse kinetic pressure forces. As mentioned above, our discussion of this topic will concentrate on idealized "zero-emittance" beams.

The first point concerns the fact that a (laminar) sheet beam with constant thickness $t$ and uniform density $n_{0}$ cannot be "matched" in the $y$ dimension (equal space charge and magnetic focusing forces for all values of $y$ in the beam) simultaneously for all points across the beam width, $-w / 2 \leqslant x \leqslant+w / 2$. This can be appreciated from the fact that the $y$ component of space-charge field decreases near the beam edge at $|x|=w / 2$ due to fringing effects. In particular, this field reduction-due to image charges in the waveguide walls-occurs within a distance $b$ of the beam edge and results in a factor of 2 reduction in $E_{y}$ as seen in Fig. 15 [calculated from Eq. (31b) for $w / b \gg 1$ ]. 


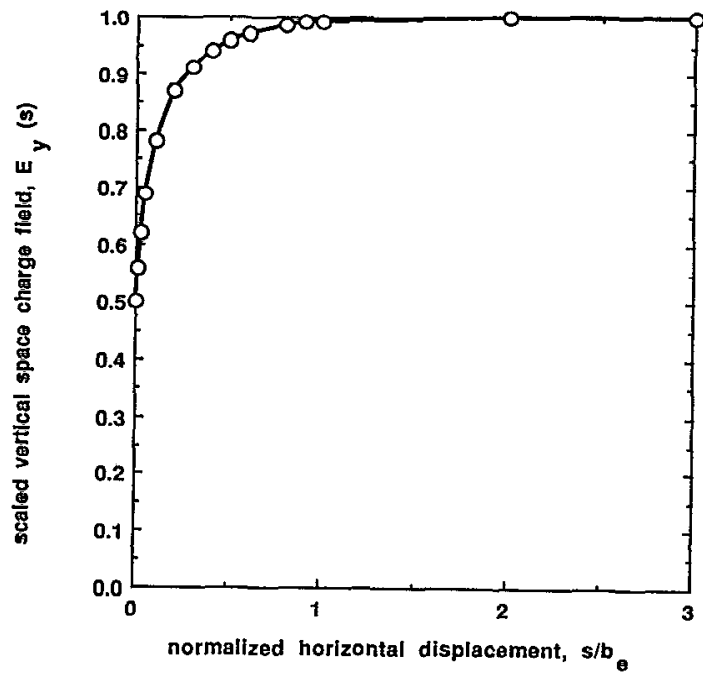

FIG. 15. Vertical space-charge-field variation across the width of a uniform density, constant thickness sheet beam confined between two parallel conducing plates separated by a distance $b_{e} s$ is a horizontal position coordinate measuring displacement relative to the beam edge (at $s=0$ ). $s=3$ corresponds to the center of the beam in this example.

Hence, setting $\Omega_{0}^{2} \geqslant 2 \omega_{p}^{2}$ to obtain beam matching in the center results in overfocusing (and probable emittance growth) in the vertical direction at the beam edges.

Next, we consider the issue of beam matching in the horizontal dimension. In general, matching over the entire beam cross section requires that defocusing and focusing forces have the same spatial dependence. This occurs naturally in the $y$ dimension since the space-charge force ( $y$ component) and periodic focusing force (near $y \approx 0$ ) both vary linearly with dimension $y$ across the beam. The same cannot be said for horizontal force components associated with a beam of constant thickness and uniform density. For example, with the closed-side magnet configuration of Fig. 7, the $x$ component of the focusing force has an approximately linear dependence on $x$, as discussed previously. From Eq. (31a), however, it is apparent that near the beam edge at $x=w / 2$, the space-charge force varies much more rapidly with $x$ (i.e., exponentially, due to the hyperbolic sine term). To remedy this functional mismatch, one is tempted to consider a beam with a diffuse, parabolic density dependence, $n(x) \approx n_{0} x^{2} / L_{n}^{2}$. According to Eq. (35a), such a density profile would yield the desired linear dependence of the space-charge field on $x$; however, while improving the matching in the $x$ dimension, this parabolic density profile will only exacerbate the previously mentioned mismatch in the $y$ dimension near the beam edges $(|x|=w / 2)$.

The situation improves slightly when one considers the open-side configuration for horizontal focusing. In Fig. 16, we have plotted the spatial dependence of horizontal focusing and defocusing forces given a sharp-edged, uniform density beam and open-side focusing [see Eqs. (31a) and (44)]. Both forces increase rapidly with $x$ as one approaches the beam edge, although the gradient scale length for the magnetic field is longer than for the space-charge

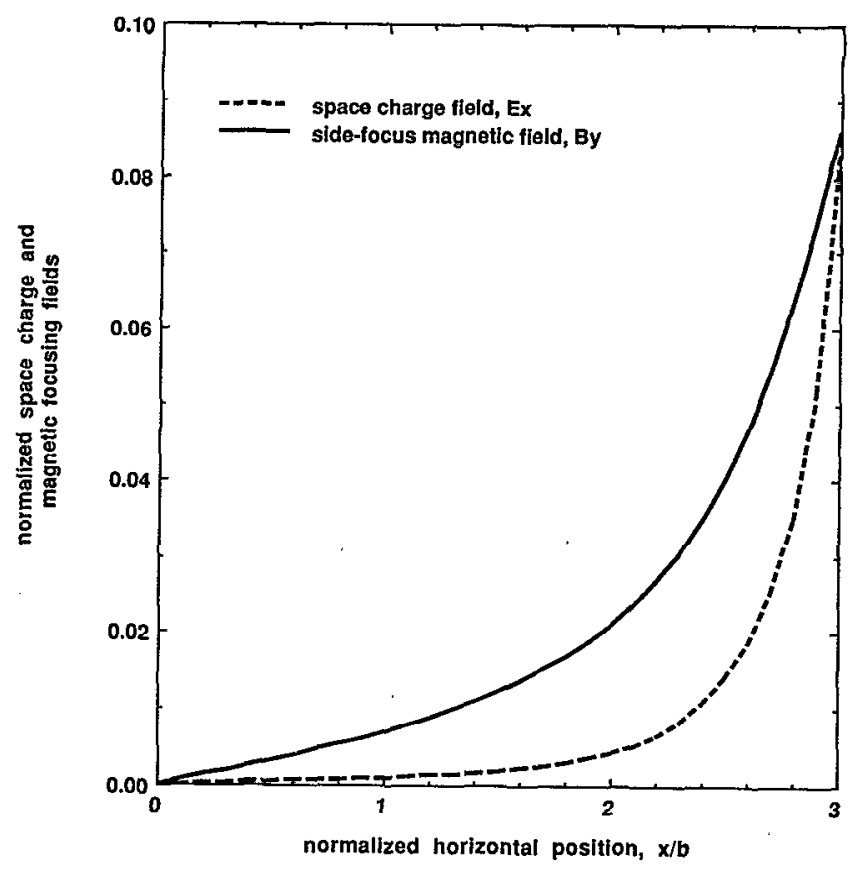

FIG. 16. Variation with $x$ of horizontal (magnetic) focusing and (space charge) defocusing forces for a sharp-edged, uniform density, constant thickness (rectangular) sheet beam in the open-side PCM configuration of Fig. 9 or Fig. 10. In this instance the waveguide wall spacing and magnet gap are equal $\left(b_{e}=b_{m}=b\right)$, and $x / b=0$ represents the beam cenlter while $x / b=3$ represents the beam edge $(t / b=0.1)$.

field. Again, one is tempted to consider profiling the beam density in the $x$ dimension to achieve more nearly equal spatial variations between the two forces. To illustrate, we consider a density profile that is parabolic for $|x| \leqslant w / 2$ but has sharp edges at $|x|=w / 2$ as pictured in Fig. 17. This particular choice is motivated by the fact that the spacecharge fields can be analytically computed from Eq. (37). For a very large density gradient scale length $L_{n}$, one obtains the previous result shown in Fig. 16. However, as illustrated in Fig. 18 for $w / b=6$, a close match in the spatial dependencies of the space charge and magnetic forces is realized with modest profiling obtained with $L_{n}$ ' $b=4$. Again, however, such profiling causes the beam density to be lower at the sides than in the beam center resulting in beam edges that are overfocused in the $y$ dimension.

The discussion above illustrates that self-consistent matching of a zero-emittance sheet beam in both dimen-

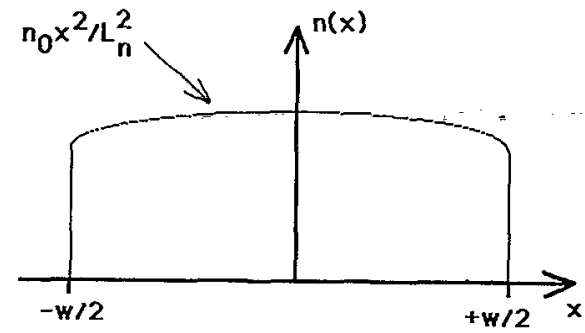

FIG. 17. Horizontal density profile with abrupt edges used for approximate match of space-charge and focusing forces in Fig. 18. 


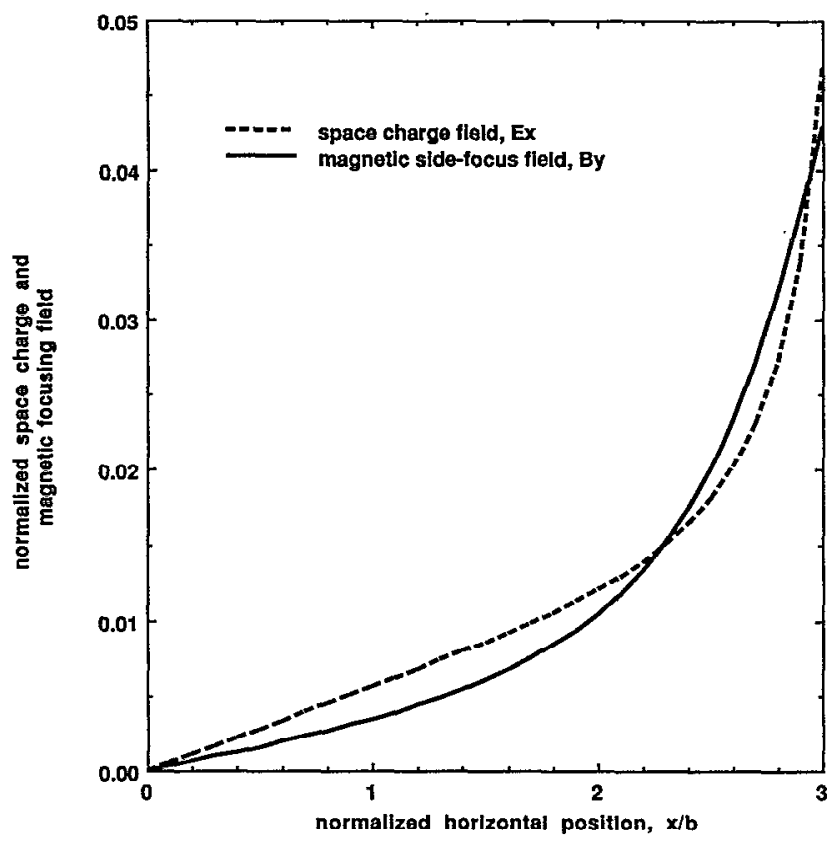

FIG. 18. Approximately matched horizontal focusing and defocusing forces for a constant thickness sheet beam having the density profile of Fig. 7 with $L_{n} / b=4$ and confined by the open-sided PCM configuration of Fig. 10. For this example, $b_{m}=b_{e}=b, w_{s}=w=6 b$, and $t / b=0.1$.

sions across the entire cross section is not achievable with constant thickness beams. In particular, the regions suffering from greatest mismatch (and therefore vulnerable to the greatest emittance growth) occur within a waveguide wall separation distance $b$ of the sheet beam edges. Complete matching of the beam (if possible), including these edge regions, would require a complicated prescription for beam density and emittance profiles-a topic for future study. On the other hand, for large values of $w / b$, these higher-emittance edge regions will constitute a small portion of the overall beam and-depending on the application-may be negligible.

\section{Matching with sheet beams by profiling the beam thickness: Elliptical sheet beams}

An alternative approach to beam matching involves profiling the thickness (modifying the beam cross-sectional shape) rather than the density. For example, based on calculations by Lapostolle, ${ }^{28}$ approximate expressions for the electric-field components due to a uniformly dense beam with elliptical cross section can be written as

$$
E_{x}=\frac{1}{\epsilon_{0}} \frac{q n r_{y}}{\left(r_{x}+r_{y}\right)} x=-\frac{m}{e} \omega_{p}^{2} \frac{r_{y}}{\left(r_{x}+r_{y}\right)} x
$$

and

$$
E_{y}=\frac{1}{\epsilon_{0}} \frac{q n r_{x}}{\left(r_{x}+r_{y}\right)} y=-\frac{m}{e} \omega_{p}^{2} \frac{r_{x}}{\left(r_{x}+r_{y}\right)} y,
$$

where $r_{x}$ and $r_{y}$ are the semiaxes of the ellipse in the horizontal and vertical transverse dimensions, respectively. Note thal these fields are linear in both $x$ and $y$, automat-

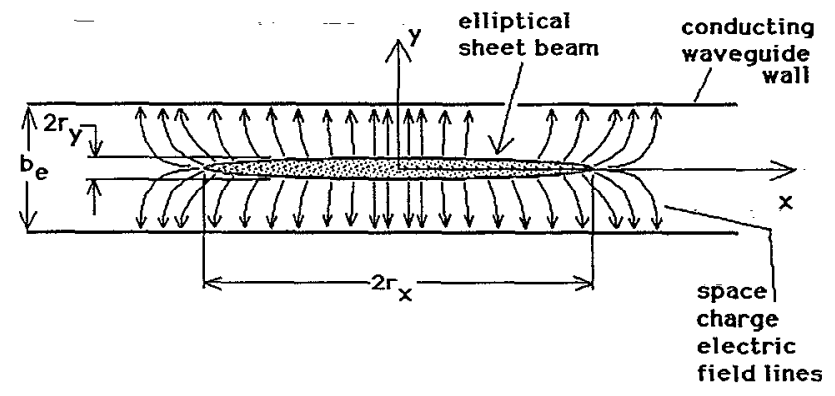

FIG. 19. Distortion of space-charge electric fields of an elliptical beam by conducting waveguide walls.

ically ensuring that a matchable condition exists with the linear forces [Eqs. (7)] obtained from closed-side, twoplane PCM focusing (see Figs. 1 and 8 ). In particular, a matched beam will be realized for

$$
\frac{\Omega_{0}^{2}}{2}=\omega_{p}^{2} \frac{r_{x}}{\left(r_{x}+r_{y}\right)}
$$

and

$$
\frac{\Omega_{0}^{2}}{2} \frac{b_{m}^{4}}{a_{m}^{4}}=\omega_{p}^{2} \frac{r_{y}}{\left(r_{x}+r_{y}\right)} .
$$

In the limit that $r_{x} \gg r_{y}$, matching is achieved by setting $\omega_{p}^{2}=\Omega_{0}^{2} / 2$ to balance forces in the vertical dimension [Eq. (54a)], followed by separately adjusting the ratios $b_{m} / a_{m}$ and $r_{x} / r_{y}$ to achicve balance in the horizontal dimension [Eq. (54b)]. Taking the example of $b_{m} / a_{m}=0.2$, this requires a very thin elliptical sheet beam with $r_{x} / r_{y}=625$.

The next issue concerns the effect of nearby conducting walls on the space-charge fields of an elliptical-crosssection sheet beam, as illustrated in Fig. 19. This is analyzed through the use of the Green's function for a beam between two parallel plates [viz., Eq. (28)] and the expressions

$$
\begin{aligned}
& E_{x}=-q n \int_{-r_{x}}^{r_{x}} d x^{\prime} \int_{-y_{b}\left(x^{\prime}\right)}^{y_{b}\left(x^{\prime}\right)} d y^{\prime} \frac{\partial G_{\|}\left(x, x^{\prime} ; y, y^{\prime}\right)}{\partial x} \\
& E_{y}=-q n \int_{-r_{x}}^{r_{x}} d x^{\prime} \int_{-y_{b}\left(x^{\prime}\right)}^{y_{b}\left(x^{\prime}\right)} d y^{\prime} \frac{\partial G_{\|}\left(x, x^{\prime} ; y, y^{\prime}\right)}{\partial y},
\end{aligned}
$$

wherc

$$
y_{b}(x)=r_{y} \sqrt{1-x^{2} / r_{x}^{2}}
$$

locates the perimeter of the elliptical-cross-section beam. Normalizing all quantities as previously done in the derivation of Eq. (39a) we obtain 


$$
\begin{aligned}
\left|E_{x}(x, y)\right|= & \sum_{j=0}^{\infty} \frac{2 \cos [(2 j+1) \pi y]}{(2 j+1) \pi}\left(\int_{-r_{x}}^{x} d x^{\prime} \sin \left[(2 j+1) \pi y_{b}\left(x^{\prime}\right)\right] e^{-(2 j+1) \pi\left(x-x^{\prime}\right)}\right. \\
& \left.-\int_{x}^{r_{x}} d x^{\prime} \sin \left[(2 j+1) \pi y_{b}\left(x^{\prime}\right)\right] e^{+(2 j+1) \pi\left(x-x^{\prime}\right)}\right)
\end{aligned}
$$

and

$$
\begin{aligned}
\left|E_{y}(x, y)\right|= & \sum_{j=0}^{\infty} \frac{2 \sin [(2 j+1) \pi y]}{(2 j+1) \pi}\left(\int_{-r_{x}}^{x} d x^{\prime} \sin \left[(2 j+1) \pi y_{b}\left(x^{\prime}\right)\right] e^{-(2 j+1) \pi\left(x-x^{\prime}\right)}\right. \\
& \left.+\int_{x}^{r_{x}} d x^{\prime} \sin \left[(2 j+1) \pi y_{b}\left(x^{\prime}\right)\right] e^{+(2 j+1) \pi\left(x-x^{\prime}\right)}\right)
\end{aligned}
$$

where (normalized) dimensions are in units of $b_{e}$ (the waveguide wall separation distance) and the fields are in units of $E_{0}=-e n_{0} b_{e} / \epsilon_{0}$.

The basic behavior of the fields is illustrated by the following numerically evaluated cases. First, we computed the horizontal field $E_{x}(x, y=0)$ as a function of (normalized) horizontal position for several representative values of waveguide wall separation. In particular, Fig. 20 displays $E_{x}(x)$ in the $y=0$ plane for $\left(r_{x} / b_{e}, r_{y} / b_{e}\right)$ $=(0.375,0.0125),(3,0.1)$, and $(6,0.2)$, respectively. For the case of distant conducting walls $(0.375,0.0125), E_{x}(x)$ has the linear dependence predicted in Eq. (53a); however, as the walls are brought closer $[(3,0.1)$ and $(6,0.2)]$, image charge shielding causes the horizontal space-charge field to increase more rapidly near the edges of the beam.

The next question is whether a beam that is matched to PCM focusing in the vertical dimension at the beam center $(x=0)$ remains matched across the entire cross section. Recall that for the constant thickness sheet beam with uni-

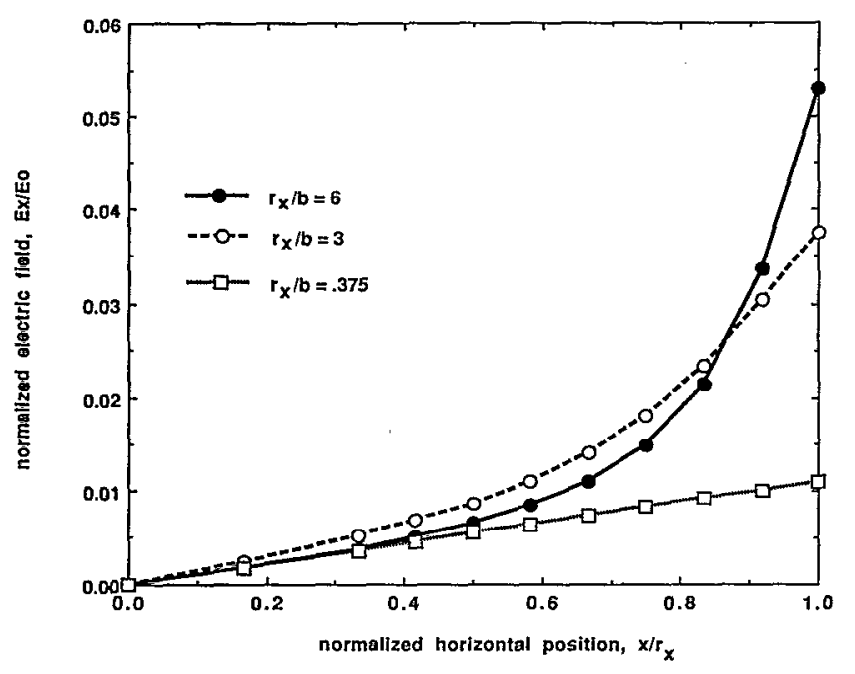

FIG. 20. Horizontal space-charge elcctric field $E_{x}(x, y=0)$ for an clliptical sheet beam between two conducting plates separated by distance $b$. $r_{x} / r_{y}=30$ for all three cases. Large $r_{x} / b$ corresponds to closely spaced conducting walls, while small $r_{x} / b$ corresponds to remotely spaced conducting walls. form density, a beam that was matched in the center near $x=0$ was overfocused at the edges near $x=w / 2$, due to a reduction in $E_{y}$ with increasing $x$ (Fig. 15). To check this for the elliptical sheet beam, we numerically evaluated $E_{y}\left(x, y=y_{b}\right)$ [viz., Eq. (55b)] for large and small waveguide wall spacing, i.e., $\left(r_{x} / b_{e}, r_{p} / b_{e}\right)=(0.375,0.0125)$, and $(6,0.2)$, respectively. To determine whether matching in the vertical direction at the beam perimeter is satisfied for all values of $x$, we compare $E_{y}\left(x, y_{b}\right)$ with $y_{b}(x)$, since the PCM focusing in the vertical plane is a linear function of $y$. The results shown in Fig. 21 indicate the desired vertical matching of forces across the entire beam width.

The results displayed in Figs. 20 and 21 suggest that two possible matched configurations are possible. In the limit of large $b_{e}$ (distant waveguide walls), the spacecharge fields are linear in both dimensions and are well matched with the linear forces of closed-side two-plane PCM focusing as discussed previously. On the other hand,

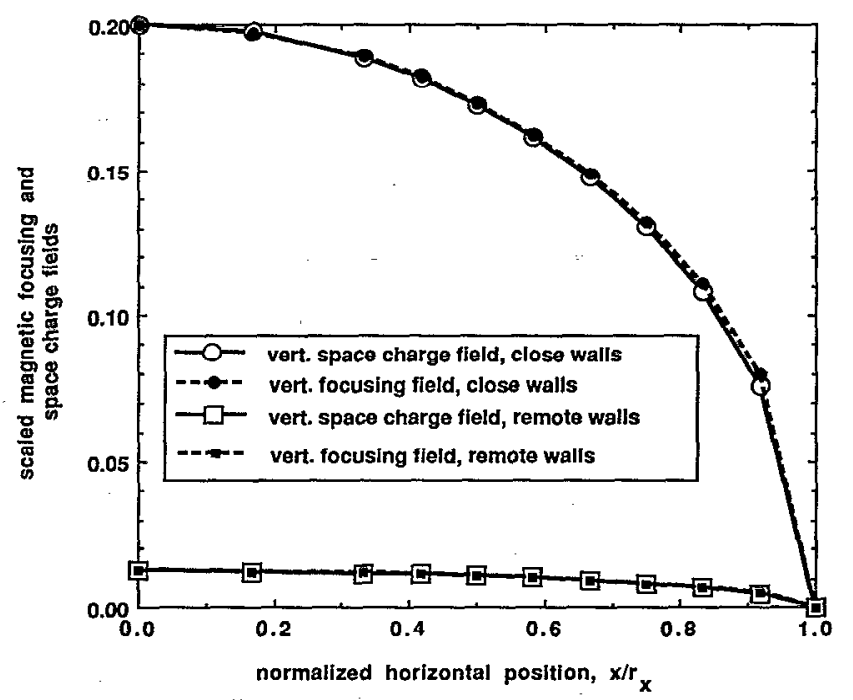

FIG. 21. Demonstration of matching between vertical space charge and vertical PCM focusing forces (at elliptical beam perimeter) across the entirc beam width, independent of whether conducting waveguide walls are in close proximity or distant. Circles (open and solid) correspond to close proximity walls $\left(r_{x} / b_{e}=6\right)$, while squares (open and solid) correspond to remotely positioned wavèguide walls $\left(r_{x} / b_{e}=0.375\right)$. In all cases, $r_{x} / r_{y}=30$. 


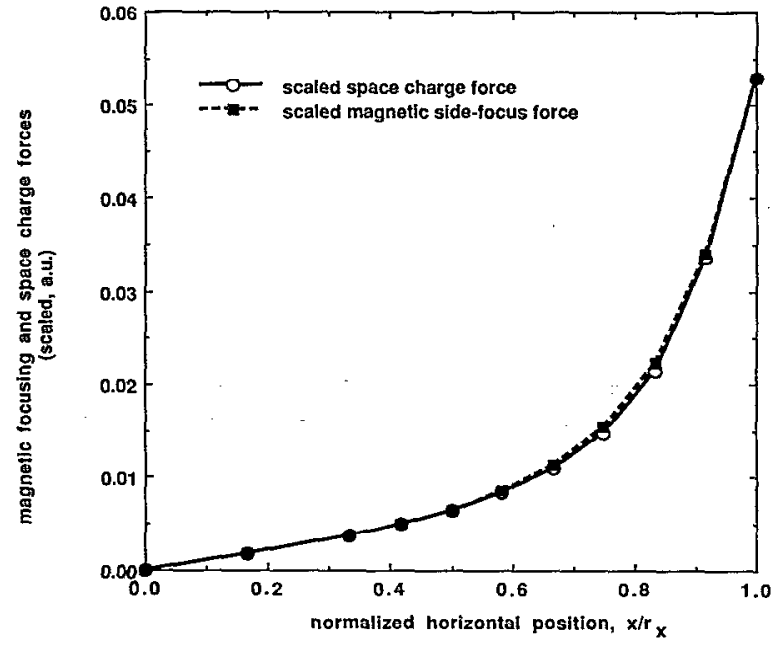

FIG. 22. Demonstration of matching between horizontal space-charge and offset-pole-piece focusing forces across entire beam width. For this example, $r_{x} / b_{e}=6, r_{y} / b_{e}=0.2, w_{s} / b_{m}=7, w_{s}=2 r_{x}$, and $b_{m}=(12 / 7) b_{e}$.

for smaller values of $b$, the space-charge fields are linear and match single-plane PCM focusing in the vertical dimension, but are nonlinear and better matched to the openside focusing configuration [viz., Fig. 9 or Fig. 10] in the horizontal dimension. That such a match is possible is illustrated in Fig. 22 where, taking $w_{s}=2 r_{x}$ we have plotted (normalized) $E_{x}$ vs $x$ for $\left(r_{x} / b_{e}, r_{y} / b_{e}\right)=(6,0.2)$ and compared it with the (normalized) open-side, horizontal focusing force for $w_{s} / b_{m}=7$ [this combination of parameters corresponds to having the magnet gap somewhat larger than the waveguide wall gap, i.e., $\left.b_{m}=(12 / 7) b_{e}\right]$. The close match between magnetic focusing and space-charge defocusing forces as a function of horizontal position is evident.

\section{SUMMARY AND CONCLUSIONS}

Sheet beams would be attractive for numerous applications, but are subject to disruptive instabilities and emittance growth. In this article we have analyzed the confinement characteristics of sheet beams focused by periodic cusped magnetic (PCM) focusing. The analysis has concentrated on nonrelativistic (low voltage) sheet beams, becausc space-charge forces are often more significant in this regime in comparison with relativistic applications. Two rectangular-cross-section magnetic configurations capable of focusing in both transverse directions were considered: (i) a closed-side two-plane PCM configuration that is topologically equivalent to conventional round-cross-section PPM focusing; and (ii) an open-side configuration that uses ponderomotive PCM focusing in the vertical plane and simple $v_{z} B_{y}$ Lorentz force focusing in the horizontal planc. Three types of sheet bcams wcre studied: (i) sheet beams with constant thickness (rectangular cross section) and uniform density; (ii) sheet beams with constant thickness (rectangular cross section) and a density variation in the horizontal plane; and (iii) sheet beams with elliptical cross section and uniform density. Electrostatic and mag- netostatic field formulas were derived for the three types of sheet beams and two magnetic configurations, respectively.

Using a simplified fluid model (first introduced by Buneman for sheet beams in solenoidal magnetic fields) we have illustrated the fact that periodically focused sheet beams (either PCM or wiggler) should be stable against low-frequency perturbations such as the diocotron mode. This stability should hold for all modes with growth lengths longer than the spatial magnetic period.

We also investigated two-plane beam focusing requirements. Period-averaged orbit dynamics in the closed-side two-plane PCM fields show that ponderomotive focusing in the narrow (vertical) transverse dimension is equal in magnitude to planar "wiggler" focusing or cylindrical PPM focusing, but focusing in the horizontal plane is much weaker-by approximately a factor of the crosssection aspect ratio to the fourth power, $\left(b_{m} / a_{m}\right)^{4}$. As a result, two-plane focusing of rectangular thickness sheet beams with uniform thickness and density appears straightforward in the vertical dimension, but problematic in the horizontal dimension. On the other hand, two-plane focusing of such beams with the open-side configuration appears to be well within the capabilities of existing magnet technology. Adding a density variation or diffuse edges (to the constant thickness sheet beam) in the horizontal dimension such that beam density is peaked near the center would tend to make focusing in the horizontal dimension more feasible for closed-side PCM focusing (by reducing space-charge fields at the beam edge), but it exacerbates beam mismatch problems near the beam edges.

Finally, we discussed the issue of beam matching in order to minimize beam envelope oscillations or emittance growth. The discussion only considered cold, or zeroemittance, beams. In general, it was illustrated that laminar sheet beams with constant thickness cannot be matched with either the closed- or open-side PCM magnet configurations. The most troublesome regions, as mentioned above, are within a waveguide wall separation distance of the beam edges in the horizontal plane. On the other hand, elliptical-cross-section sheet beams with uniform density can be matched with either the open- or closed-side PCM configurations by suitable adjustments to magnet, beam, and waveguide cross-section dimensions. Of the two magnet alternatives, matching in the closed-side two-plane PCM configuration may be more challenging due to the large eccentricity or aspect ratio $\left(r_{x} / r_{y} \gg 1\right)$ required in the beam's elliptical cross section for reasonable choices of magnet dimensions. This large elliptical aspect ratio is a result of the significantly weaker focusing force in the horizontal plane of a closed-side two-plane PCM configuration. Fabrication of a beam source with the precise elliptical cross section may be difficult when such a large aspect ratio is required. On the other hand, fabrication of elliptical cross section beam sources that are matched to open-side PCM focusing appears much more practical.

\section{ACKNOWLEDGMENTS}

The authors would like to acknowledge helpful discussions with Dr. S. Bidwell, Dr. S. F. Chang, Dr. V. L. 
Granatstein, Dr. B. Levush, Dr. J. E. Scharer, and Dr. R. True. Appreciation is also expressed to J. Joe, J. Anderson, and D. Hass for contributions to the magnetic-field calculations as well as assistance with some of the figures. This work was supported in part by the DoD Vacuum Electronics Initiative as managed by the Air Force Office of Scientific Research (Grant No. AFOSR-91-0381) and by a National Science Foundation Presidential Young Investigator Award (ECS-9057675).

${ }^{1}$ J. H. Booske, W. W. Destler, Z. Segalov, D. J. Radack, E. T. Rosenbury, J. Rodgers, T. M. Antonsen, Jr., V. L. Granatstein, and I. D. Mayergoyz, J. Appl. Phys. 64, 6 (1988).

${ }^{2}$ V. A. Lebedev, I. N. Meshkov, and A. N. Sharapa, Sov. Phys. Tech. Phys. 24, 421 (1979).

${ }^{3}$ J. R. Pierce, IRE Trans. Electron Devices ED-3, 183 (1956).

${ }^{4}$ R. L. Kyhl and H. F. Webster, IRE Trans. Electron Devices ED-3, 172 (1956).

${ }^{5}$ T. M. Antonsen, Jr. and E. Ott, Phys. Fluids 18, 1197 (1975), and references contained therein.

'R. C. Davidson, H. W. Chan, C. Chan, and S. Lund, Rev. Mod. Phys. 63, 341 (1991).

${ }^{7}$ S. F. Chang, J. E. Scharer, and J. H. Booske, IEEE Trans. Plasma Sci. Fourth Special Issue on High Power Microwaves PS-20, 293 (1992).

${ }^{8}$ H. C. Chen, IEEE Trans. Nucl. Sci. 32, 2380 (1985).

${ }^{9}$ R. C. Davidson, K. T. Tsang, and H. S. Hum, Phys. Fluids 31, 1727 (1988).

${ }^{10} \mathrm{G}$. Providakes, J. A. Nation, and M. E. Read, IEEE Trans. Microwave Theory Tech. MTT-25, 563 (1977).

${ }^{11}$ M. E. Read, A. J. Dudas, J. J. Petillo, and M. Q. Tran, in 14th International Conference on Infrared and Millimeter Waves, Wurzburg, Germany (SPIE, Bellingham, WA, 1989), Conf. Dig. Vol. 1240, pp. 79-80.
${ }^{12}$ J. M. Butler, R: W. Schumacher, D. M. Goebel, J. Hyman, J. Santoru, R. M. Watkins, R. J. Harvey, and F. A. Dolezal, Bull. Am. Phys. Soc. 36, 2469 (1991).

${ }^{13}$ J. H. Booske, D. J. Radack, T. M. Antonsen, Jr., S. W. Bidwell, Y. Carmel, W. W. Destler, H. P. Freund, V. L. Granatstein, P. E. Latham, B. Levush, I. D. Mayergoyz, and A. Serbeto, IEEE Trans. Plasma Sci. 18, 399 (1990).

${ }^{14}$ D. J. Radack, J. H. Booske, Y. Carmel, and W. W. Destler, Appl. Phys. Lett. 55, 2069 (1989).

${ }^{15}$ S. W. Bidwell, Z. X. Zhang, T. M. Antonsen, Jr., W. W. Destler, V. L. Granatstein, B. Levush, and J. Rodgers, in Proceedings of 9th International Conference on High Power Charged Particle Beams, Washington, DC, May 25-29, 1992 (to be published).

${ }^{16}$ R. True, IEEE Trans. Electron Devices ED-31, 353 (1984).

${ }^{17}$ G. Dohler, IEEE Trans. Electron Devices ED-28, 602 (1981).

${ }^{18} \mathrm{~J}$. D. Lawson, The Physics of Charged-Particle Beams (Clarendon, Oxford, 1977).

${ }^{19} \mathrm{~J}$. W. Gibbs, The Collected Works, Vol. 1, Thermodynamics (Yale University Press, New Haven, CT, 1948), pp. 55-62.

${ }^{20}$ O. Buneman, J. Electron. Control 3, 507 (1957).

${ }^{21}$ M. Reiser, J. Appl. Phys. 70, 1919 (1991).

${ }^{22}$ E. Esarey, W. Marable, and C. M. Tang, J. Appl. Phys. 67, 2210 (1990).

${ }^{23}$ T. C. Marshall, Free Electron Lasers (MacMillan, New York, 1985).

${ }^{24}$ E. T. Scharlemann, J. Appl. Phys. 58, 2134 (1985).

${ }^{25}$ K. E. Robinson and D. C. Quimby, in Proceedings of the 1987 IEEE Particle Accelerator Conference, March 16-19, Washington, DC (IEEE, New Jersey, 1987), pp. 428-430.

${ }^{26}$ W. W. Destler, V. L. Granatstein, I. D. Mayergoyz, and Z. Segalov, J. Appl. Phys. 60, 521 (1986).

${ }^{27}$ R. P. Walker, Nucl. Instrum. Methods Phys. Res. A 237, 366 (1988), and references cited therein.

${ }^{28}$ P. M. Lapostolle, CERN report AR/Int. SG/65-15, Geneva, Switzerland, July 1965 (unpublished). 